NISTIR 6275

\title{
The Molecular Level Design of Fire Retardants and Suppressants
}

Marc R. Nyden

Building and Fire Research Laboratory

Gaithersburg, Maryland 20899

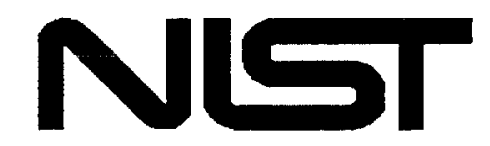

United States Department of Commerce

Technology Administration

National Institute of Standards and Technology 


\section{The Molecular Level Design of Fire Retardants and Suppressants}

Marc R. Nyden

January 1999

Building and Fire Research Laboratory

National Institute of Standards and Technology

Gaithersburg, MD 20899

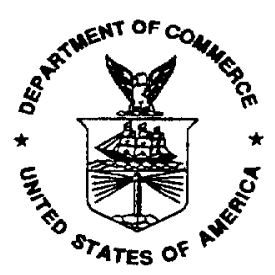

U.S.Department of Commerce

William M. Daley, Secretary

Technology Administration

Gary R. Bachula, Acting Under Secretary for Technology

National Institute of Standards and Technology

Raymond G. Kammer, Director 


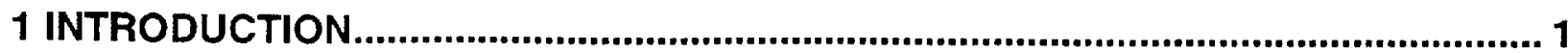

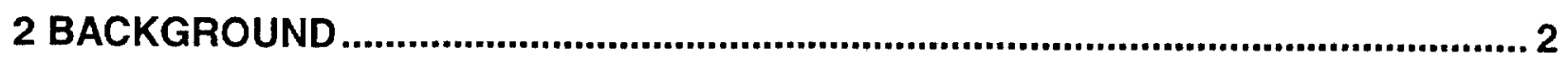

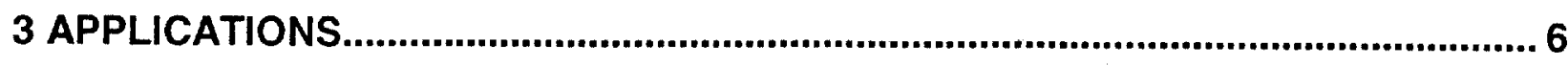

3.1 Atmospheric Lifetimes of Halon Replacement Candidates ...............................................................................6 6

3.2 Molecular Modeling of the Thermal Degradation of Polymers......................................................................... 11

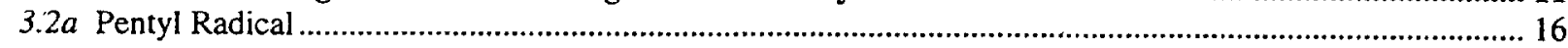

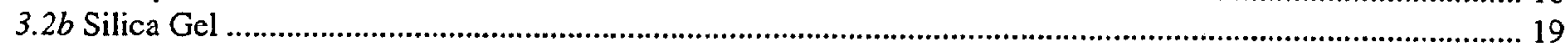

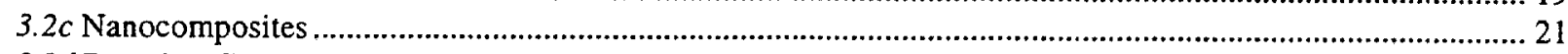

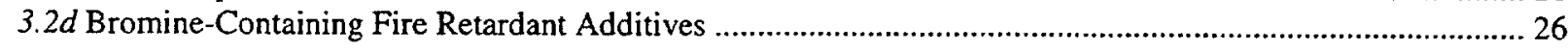

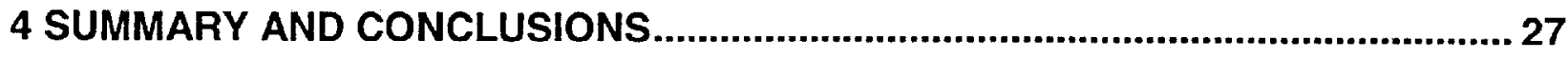

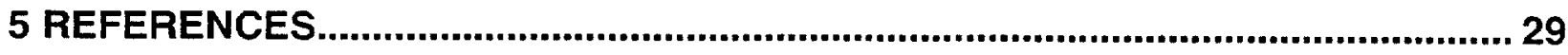




\section{Acknowledgements}

MD_REACT was developed as part of a Cooperative Research and Development Agreement between NIST and MSI (CRADA: CN-1241). Partial support for this work was provided by the Federal Aviation Administration under Interagency Agreement DTFA003-92-Z-0018 monitored by Dr. Richard E. Lyon and from the Industrial Consortium for Environmentally Friendly Flame Retardants.

The author is grateful to Dr. Wing Tsang (NIST) for suggesting the kinetic study of pentyl radical.

Abstract
The various methods that comprise the field of molecular modeling are reviewed in the context of their potential for application to the molecular level design of new fire retardants and suppressants. The capabilities of these techniques are demonstrated by performing calculations on systems ranging in molecular complexity from small gas phase molecules to polymers. As a consequence of its fundamental basis, molecular modeling can be used to predict the properties of new materials in ways that macroscopic models cannot and, thereby, offers an unrivaled potential for computer aided molecular design. Unfortunately, the amount of computational effort required to obtain this level detail currently restricts the domain of these calculations to infinitesimal scales of time and distance, which limits their application to model systems. These boundaries, however, are receding as advances in computer technology enable calculations on increasingly more complex systems. Based on the rapid pace of the progress attained so far, it seems likely that molecular modeling will soon become an integral part of industrial research and development programs in materials flammability. The purpose of this chapter is, therefore, to provide a meaningful introduction to the field with an emphasis on how these teqhniques can be applied to the design and development of fire retardants and suppressants. 


\section{Introduction}

The flammability of materials is an important concern that must addressed before they can be used in buildings, ships, aircraft and clothing. However, the present generation of fire retardant additives and fire resistant polymers has come under increasing public scrutiny because of the perception that they may contribute to environmental problems $[1,2]$. Some materials, more specifically those containing chlorinated or brominated additives and polymers, have been implicated in studies that suggest that they contribute to the formation of dioxins and furans during incineration [2]. This concern has spurred interest in the development of new and more "environmentally friendly" treatments to reduce polymer flammability.

The emergence of this issue has occurred at a time when computer technology has opened new possibilities for the molecular level design of additives and materials. Indeed, molecular modeling has become an integral part of the research and development process in many important industries. This, in turn, has spurred the growth of a new sector in the economy comprised of a multitude of small businesses that develop and support molecular modeling software [3]. Pharmaceutical companies, in particular, have pioneered the application of these techniques to the computer-aided design of biologically active compounds [4]. Recent work conducted at the Building and Fire Research Laboratory of the National Institutes of Standards and Technology has also demonstrated that molecular modeling is a promising approach to the development of new fire retardant additives and fire resistant materials [5-9].

Molecular modeling offers some potential advantages over macroscopic approaches, such as those based on chemical kinetics [10] and fluid dynamics [11], which may be used to obtain similar information about the properties of materials. These derive from the nature of the potential energy functions or forcefields (vide infra) that are the basis of most molecular models, which are generally applicable to an entire class of chemically related compounds. Thus, for example, the parameters that describe the C-C and $\mathrm{C}-\mathrm{H}$ bonds in ethane are, to a good approximation, transferable to many other hydrocarbon molecules, including polymers. On the other hand, the implementation of kinetic and/or transport models requires specific information about reaction mechanisms, rate constants, and viscosities that are not generally available. This is especially true when the purpose of the investigation is to predict the properties of hypothetical materials, for which there are no experimental data. Furthermore, the capability to examine a process at a molecular level can provide the basis for a deeper understanding of the operant mechanisms. As a consequence of this generality, molecular modeling can be used to predict the properties of new materials in ways that macroscopic models cannot, and thereby offers an unrivaled potential for computer-aided molecular design. On the other 
hand, the capability to study structure and reactivity at the molecular level of detail does not come without cost. The computational effort involved in performing simulations on polymers can be prohibitive and often restricts the scope of the investigation to infinitesimal times and distances, which may not always provide an adequate representation of the processes of interest. For this reason, molecular modeling is, at least for the time being, a complementary approach that must be combined with experimental measurements and macroscopic modeling to provide a complete picture of polymer flammability and fire resistance.

In this chapter, the various methods that comprise the field of molecular modeling will be reviewed and the viability of using these techniques to identify factors which alter the thermal degradation chemistries of polymers in ways that effect a reduction in their flammability will be assessed. The purview of this exposition will be extended to include consideration of quantum mechanical calculations performed on a series of small gas phase molecules that were advanced as candidates for the replacement of halon fire extinguishants [12]. These calculations were performed to assess the potential environmental impacts of these chemicals. This topic is included in this chapter in order to provide a relevant example of the application of molecular quantum mechanics, which at the time of this writing, is still limited to relatively small molecules.

\section{Background}

The basis of molecular modeling is the Schrödinger equation. This is a complicated partial differential equation that formally couples all of the molecular degrees of freedom. It is written in abbreviated form in Eq.(1),

$$
H(R, r) \Psi(R, r)=E \Psi(R, r),
$$

where $\mathrm{R}$ and $\mathrm{r}$ are used to denote the collective coordinates of the nuclei (i.e., atomic centers) and electrons, respectively. The Schrödinger equation is an example of, what is known in Mathematics, as an eigenvalue problem. The eigenvalue, $\mathrm{E}$, corresponds to the energy and the eigenvector, $\Psi(\mathrm{R}, \mathrm{r})$, to the wavefunction of the molecule. The importance of the wavefunction is that it provides information about the electronic structure and geometry of the molecule. Thus, the square of the wavefunction gives the probability of finding the nuclei and the electrons at the positions specified by $R$ and $r$, respectively. Eigenvalue equations are solved by standard mathematical techniques [13]. In the case of the Schrödinger equation, the wavefunction and energy are obtained by diagonalizing the Hamiltonian $(\mathrm{H})$ in the space spanned by a basis set. Extremely large basis sets are required to obtain chemical accuracy for energy-related properties in all but the smallest molecules.

The molecular Hamiltonian is the quantum mechanical operator associated with energy. It consists of Laplacian operators for each of the electrons and nuclei that 
comprise the constituent atoms and multiplicative terms that correspond to the kinetic and potential energy of the molecule, respectively. When acting on the wavefunction, it gives the total energy of the molecule in accordance with Eq.( 2 ),

$$
\langle\Psi|H| \Psi\rangle=E,
$$

where the brackets are used to denote integration over all space and

$$
\Psi(r, R)=\sum_{i}^{N C} C_{i} \Phi_{i}(r, R) .
$$

The coefficients, $C_{\mathrm{i}}$, are determined by the condition that the energy is a minimum, subject to the constraint that $\langle\Psi \mid \Psi\rangle=1$. This procedure, which is equivalent to diagonalizing the Hamiltonian in the space spanned by the NC basis functions, is justified by the variational principle, which asserts that the energy of a trial wavefunction is always greater the true ground state energy [14]. The many-body basis functions, $\Phi_{i}(r, R)$, are called configurations and Eq.( 3 ) is the configuration interaction (CI) expansion of the wavefunction.

The wavefunction in Eq. (1) can be factored into functions that independently describe the electronic structure and the thermal motions of the nuclei. This follows from the Born - Oppenheimer approximation which asserts that electrons, because they are three orders of magnitude lighter, can adjust instantaneously to changes in the positions of the nuclei [15]. The effect of separating the nuclear and electronic coordinates is represented in Eq. ( 4 ),

$$
\Psi(R, r) \approx \phi(R) \psi(r ; R)
$$

where $\psi(r ; R)$ and $\phi(R)$ are wavefunctions for the electrons and the nuclei, which may be obtained from the solution of Eqs.( 5 ) and ( 6 ), respectively.

$$
\begin{gathered}
H(r ; R) \psi(r ; R)=E_{e}(R) \psi(r ; R) \\
H\left(R, E_{e}(R)\right) \phi(R)=E \phi(R)
\end{gathered}
$$

The semicolon in Eq. ( 5 ) is used to indicate that the electronic wavefunction can be solved for a fixed nuclear framework specified by R. In this context, the electronic wavefunction is said to depend parametrically on the geometry of the molecule. The Hamiltonian for the nuclear Schrödinger equation depends explicitly on the electronic energy, $E_{e}(R)$, obtained from Eq. $(5)$. The interpretation is that the nuclei move in the potential field of the electrons. The eigenvalue, E, in Eq.( 6 ) represents the total molecular energy. This includes the kinetic energy associated with molecular rotations and vibrations, as well as, the electronic energy at the specified geometry. 
The simplicity of the notation used in Eq. ( 5 ) is deceptive. In fact, this equation can be solved exactly (i.e.,there exists an analytical solution) only in the special case of a one electron system, such as $\mathrm{H}_{2}{ }^{+}$. Thus, despite the mathematical rigor inherent in the formulation of the theory, the implementation of molecular quantum mechanics almost always involves approximations. The accuracy of the energy and other molecular properties are detcrmined by the completeness of the basis sets used in the expansion of the wavefunction. This is a two-fold problem. The many-body basis functions in the CI expansion of the wavefunction presented in Eq. ( 3 ), are products of one-body functions (orbitals) which are also expanded in a basis set. The level of accuracy obtained from a quantum mechanical calculation is dependent on the size and quality of both the $\mathrm{CI}$ and orbital basis sets. Thus, an increase in accuracy can only be attained at the expense of a concomitant increase in computer time. Indeed, the computational effort involved in performing a full CI expansion, which in principle is the exact solution of the Schrödinger equation in the space spanned by the basis set, increases as the number of one-electron basis functions (NBF) to the number of electrons (n) power.

Recent advances in computer technology and in the development of approximate methods, however, have now made it possible to calculate the properties of small gas phase molecules, some of which are of major importance to the chemical industry, to chemical accuracy. This assertion will be supported using examples of bond energy calculations to estimate the atmospheric lifetimes of candidate compounds for the replacement of halon fire extinguishants in the following section. Based on the analysis presented in the preceding paragraph, it may be inferred that the limiting factor in the application of molecular quantum mechanics is the number of electrons in the molecule. Not surprisingly, the larger the molecule the more computer time is required for the calculation. At the time of this writing, it is exceedingly difficult to obtain chemically accurate information for molecules that contain more than about 10 carbon atoms [16]. The boundaries to calculations on more complex systems, however, are receding rapidly as computers and computational methods, become increasingly more powerful.

The problem of determining molecular structure, which is the subject of Eq.( 6 ), is amenable to much greater simplifications. Indeed, atoms have sufficient mass that it is usually a good approximation to calculate their positions from classical, rather than quantum mechanical formulas. To the extent that this is true, Eq. (6) may be replaced by Newton's equations of motion,

$$
F_{i}=-\frac{\partial V\left(q_{1}, q_{2}, \ldots, q_{3 N}\right)}{\partial q_{i}}=\frac{\partial p_{i}}{\partial t},
$$

where $p_{i}$ and $q_{i}$ denote the Cartesian components of momentum and position of the $N$ atoms and $V\left(q_{1}, q_{2}, \ldots, q_{3 N}\right)=E_{e}(R)$ is the potential energy of the molecule. When transformed to Hamiltonian form, Eq. ( 7 ) becomes 


$$
\begin{aligned}
& \frac{\partial H}{\partial p_{i}}=\frac{d q_{i}}{d t}, \\
& \frac{\partial H}{\partial q_{i}}=-\frac{d p_{i}}{d t} .
\end{aligned}
$$

The classical Hamiltonian is obtained by replacing the derivative terms in the quantum mechanical operator by the classical formula for the kinetic energies of the nuclei. This result is given in Eq. $(9$ ).

$$
H=\sum_{i}^{3 N} \frac{p_{i}^{2}}{2 m_{i}}+V\left(q_{1}, q_{2}, \ldots, q_{3 N}\right)
$$

The next step in the simplification of the problem is to represent the electronic energy by introducing functions that account for the potential energy associated with the relative motions of the atomic centers. In the parlance of molecular modeling, these functions are collectively referred to as a forcefield. The source of this misnomer is that the forces that affect the motion of the atoms are calculated as the negative of the gradient of these functions, as indicated in Eq. 7 ). The forcefield is parameterized using data obtained from experimental measurements and/or quantum mechanical calculations on representative compounds [17]. In principle, the result of this process is a generic potential energy function that can be used to describe an entire class of compounds.

There are several types of calculations that are routinely performed using forcefields. Molecular mechanics is one of these. The objective of these calculations is usually to determine the geometry of a molecule by finding the coordinates that correspond to the minimum potential energy at absolute zero. The effect of temperature is taken into account in molecular dynamics, which consists of solving the equations of motion that are represented in Eq. 7 ), or equivalently, in Eq.( 8 ). Yet another type of calculation that is frequently used in conjunction with forcefields is the Monte Carlo method. This is a statistical approach, whereby the equilibrium properties of molecules, which correspond to ensemble averages, can be computed by sampling the conformational space. The relative importance of a random change in molecular geometry is commonly determined by the criterion proposed by Metropolis et al. [18]. In this method, the conformation of the molecule is allowed to change with a probability that is proportional to the Boltzmann factor, $\exp \left(-\Delta V / k_{B} T\right)$. In this expression, $\Delta V$ is the change in potential energy calculated from the forcefield resulting from the move, $\mathrm{k}_{\mathrm{B}}$ is Boltzmann's constant, and $\mathrm{T}$ is the absolute temperature. A related method based on Monte Carlo sampling of experimental molecular mass distributions, as opposed to a forcefield, has been used to elucidate the mechanisms of thermal degradation in poly- $\alpha$ methylstyrene [19] and polystyrene[20]. 
The model of thermal reactivity described in Section 13.3 .2 is based on the Centralized Valence Forcefield (CVFF) [21], that is summarized in Eq.( 10 ).

$$
\begin{gathered}
V\left(q_{1}, q_{2}, \ldots, q_{3 N}\right)=\sum_{i j}^{n b o n d s} V_{b}\left(r_{i j}\right) \\
+\sum_{i j k}^{\text {nangles }} V_{a}\left(\theta_{i j k}\right) \\
+\sum_{i j k l}^{\text {norsions }} V_{t}\left(\phi_{i j k l}\right)+\sum_{i j}^{\text {npairs }} V_{n b}\left(r_{i j}\right)
\end{gathered}
$$

The first term on the right hand side of this equation represents the kinetic energy of the $\mathrm{N}$ atoms in the model polymer. The next terms are the potential energies for bond stretching $\left(\mathrm{V}_{\mathrm{b}}\right)$, bending $\left(\mathrm{V}_{\mathrm{a}}\right)$, and a torsional potential $\left(\mathrm{V}_{\mathrm{t}}\right)$ that restricts internal rotation around the covalent bonds. The last term represents the nonbond potential energy $\left(\mathrm{V}_{\mathrm{nb}}\right)$ resulting from the interactions between pairs of atoms that are not covalently bonded to each other.

\section{Applications}

\section{I Atmospheric Lifetimes of Halon Replacement Candidates}

Halons $1301\left(\mathrm{CF}_{3} \mathrm{Br}\right)$ and $1211\left(\mathrm{CF}_{2} \mathrm{ClBr}\right)$ have been the fire-fighting agents of choice since the early $1950 \mathrm{~s}$. The commercial production of these chemicals was banned by international agreement in 1994 because of their potential for destroying stratospheric ozone [22]. In recent years, considerable effort has been directed to the search for suitable replacement chemicals [23-25]. To be acceptable as a candidate, the environmental impact of a chemical must be demonstrably less than that of the halons it is supposed to replace. Atmospheric lifetime, which is the average length of time it will take before the compound is removed from the atmosphere by natural processes (e.g. rain out of oxidized products), is a reliable predictor of both ozone depletion potential (ODP) and global warming potential (GWP) [23].

The principal atmospheric removal mechanism for trace compounds that have an abstractable $\mathrm{H}$ is reaction with $\mathrm{OH}$ radical in the troposphere [23]. Reliable assessments of $\mathrm{OH}$ reactivity can be made on the basis of kinetic measurements [23]. This process, however, can be tedious and expensive because painstaking efforts have to be taken to ensure sample purity. The presence of even trace amounts of a reactive impurity will invalidate the results [26]. The prospect of being able to estimate the atmospheric lifetime of a replacement candidate on the basis of quantum mechanical calculations is, therefore, very appealing.

There is good reason to believe that this is a viable strategy. The rate-determining step for the reaction with $\mathrm{OH}$, at least for saturated halocarbons, is the abstraction of the 
$\mathrm{H}$ by $\mathrm{OH}$, which requires breaking a $\mathrm{C}-\mathrm{H}$ bond. On this basis, it seems plausible that the rate should correlate with the strength of the weakest $\mathrm{C}-\mathrm{H}$ bond in the molecule. Indeed, according to Ahrrenius theory, the rate constant depends exponentially on the activation energy for the reaction $\left(E_{a}\right)$ which should, in the case of $\mathrm{H}$ abstraction, be proportional to the amount of energy required to break the bond. On this basis, we would expect a linear relationship between the logarithm of the rate constants for the reaction of the candidate molecules $\left(\mathrm{k}_{\mathrm{C}}\right)$ and the bond dissociation energies of the reacting bonds $\left(\mathrm{D}_{\mathrm{C}-\mathrm{H}}\right)$.

Furthermore, assuming that the concentration of $\mathrm{OH}$ is essentially constant (i.e., that the reaction is psuedo first order in $\mathrm{RX}-\mathrm{H}$ ), the atmospheric lifetime of the replacement candidate $(\mathrm{C})$ should be proportional to $\mathrm{k}_{\mathrm{C}}{ }^{-1}$. The following formula, which is based on the well-established rate constant and lifetime of methyl chloroform $\left(\mathrm{CH}_{3} \mathrm{CCl}_{3}\right)$, is widely used to estimate the lifetimes of halocarbons in-lieu of detailed atmospheric modeling:

$$
\tau=7.0 \frac{k_{M C}(277)}{k_{C}(277)} .
$$

In Eq. ( 11$) \tau$ is the tropospheric lifetime of the candidate in years, $\mathrm{k}_{\mathrm{MC}}(277)$ is the $\mathrm{OH}$ rate constant for methyl chloroform at $277 \mathrm{~K}$ (a value which, when used in this context, is found to give good agreement with atmospheric models) which has a lifetime in the troposphere of about 7.0 years [27]. Substitution of the Ahrrenius expression for $\mathrm{k}_{\mathrm{C}}$ and the presumed linear dependence of $\mathrm{E}_{\mathrm{a}}$ on $\mathrm{D}_{\mathrm{C}-\mathrm{H}}$ gives, after taking the natural logarithm of both sides, Eq.( 12 ).

$$
\ln \tau=a D_{C-H}+b
$$

The slope (a) and intercept (b), which depend on the relationship between $E_{a}$ and $D_{C-H}$, are assumed to be constant for a class of related compounds. The validity of this equation is confirmed in Figure 1, which is a plot of $\ln \tau$ [28] vs $D_{\mathrm{C}-\mathrm{H}}$ [29] for a series of hydrofluorocarbons.

Although Eq.( 12 ) makes intuitive sense, it does not provide quantitative accuracy for the prediction of atmospheric lifetimes. This is a consequence of the exponential dependence of $\tau$ on $\mathrm{D}_{\mathrm{C}-\mathrm{H}}$, which has the effect of magnifying errors in the bond dissociation energies. Thus, the uncertainties in $\tau$ based on the linear fit to $\ln \tau$ illustrated in Figure 1 are on the order of about $35 \%$ of the predicted values. This estimate was derived from the average residual error and presumably reflects experimental errors in the bond energies and lifetimes, as well as weaknesses in the assumptions used in the derivation of Eq. ( 12 ). Nevertheless, in the absence of a more quantitative relationship, Eq. ( 12 ) does provide the capability to make qualitative assessments of atmospheric impact and should, therefore, be useful as a tool for screening replacement candidates. What follows is a brief description of quantum mechanical methods that can be used to calculate bond dissociation energies and the values obtained for a series of hydrofluorocarbons and hydrochlorocarbons. 


\section{Atmospheric Lifetime Vs C-H Bond Dissociation Energy}

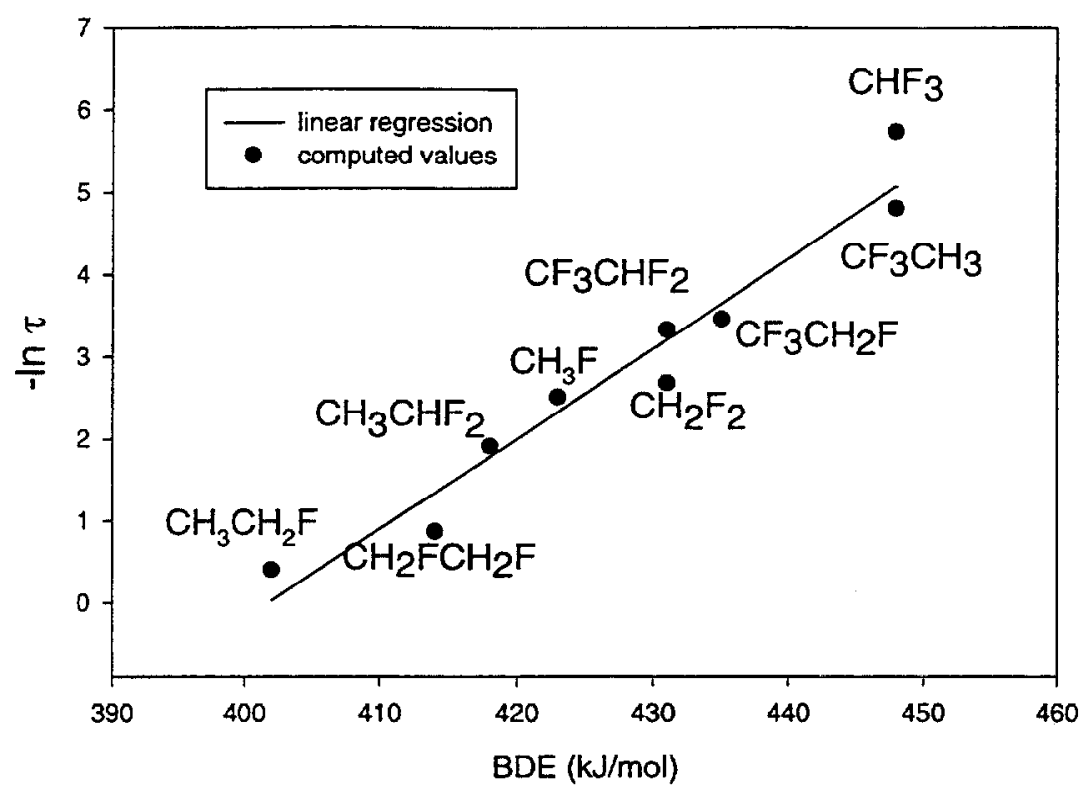

Figure 1. The natural logarithm of the tropospheric lifetimes (in years) of a series of fluoro-methanes and ethanes from Eq.(11) plotted as a function of the dissociation energy of the reacting $\mathrm{C}-\mathrm{H}$ bond (circles).

The bond dissociation energies were calculated directly as the difference in energy between the radical that results from breaking the bond of interest and the molecule. Some of the substituted methanes, which are the focus of this study, have multiple $\mathrm{C}-\mathrm{H}$ bonds. Although this could potentially introduce an ambiguity, it turns out that in all cases under consideration, the $\mathrm{C}-\mathrm{H}$ bonds in any given molecule are equivalent by symmetry. Thus, it was only necessary to perform two calculations on each molecule (i.e., one on the molecule and one on the radical) to obtain the full set of $\mathrm{C}-\mathrm{H}$ bond dissociation energies.

The results obtained from a series of quantum mechanical methods are listed in the columns of Table I from left to right in order of increasing computational effort of the method. The first three entries, corresponding to the local density functional approximation with exchange only (LDA) [30], the VWN (Vosko, Wilk and Nusair) density functional with both exchange and correlation [30], and the Hartree Fock (HF) method [31], are based on single configuration wavefunctions (i.e., $\mathrm{NC}=1$ ) constructed from energy optimized orbitals. The feature that distinguishes these calculations is their treatment of electron repulsion, which is the most complicated term in the Hamiltonian, in the equations used to determine the orbitals. Thus, in the case of the density functional methods, the potential due to the repulsion of the electrons is approximated by a simple 
function of the electron density that is justified by analogy to the exact expression for a uniform electron gas [32]. The VWN method is a variant of the LDA that includes an additional component to account for the effect of electron correlation, which is the dynamic coupling between electrons resulting from their mutual repulsion, on the orbitals.

Table I. C-H Bond Dissociation Energies in the Fluoro- and Chloro-Methanes (kJ/mol)

\begin{tabular}{ccccccc}
\hline Molecule & $\mathrm{LDA}^{\mathrm{a}}$ & $\mathrm{VWN}^{\mathrm{a}}$ & $\mathrm{HF}^{\mathrm{b}}$ & $\mathrm{MP}^{\mathrm{b}}$ & $\mathrm{G2}^{\mathrm{b}}$ & Experiment $^{\mathrm{c}}$ \\
\hline $\mathrm{CH}_{3}-\mathrm{H}$ & 494 & 464 & 362 & 458 & 435 & 440 \\
$\mathrm{CFH}_{2}-\mathrm{H}$ & 464 & 447 & 353 & 440 & 424 & 424 \\
$\mathrm{CF}_{2} \mathrm{H}-\mathrm{H}$ & 458 & 496 & 354 & 441 & 424 & 432 \\
$\mathrm{CF}_{3}-\mathrm{H}$ & 482 & 461 & 373 & 462 & 445 & 447 \\
$\mathrm{CClH}_{2}-\mathrm{H}$ & 465 & 444 & 351 & 418 & 416 & 422 \\
$\mathrm{CCl}_{2} \mathrm{H}-\mathrm{H}$ & 431 & 419 & 338 & 401 & 401 & 414 \\
$\mathrm{CCl}_{3}-\mathrm{H}$ & 415 & 401 & 322 & 385 & 390 & 401 \\
\hline
\end{tabular}

a. Reference 30 .

b. Reference 31 .

c. Reference 29 .

This effect is ignored in the HF method, where the electron in each orbital experiences only the average electron repulsion of the n-1 remaining electrons. Consequently, the bond energies obtained from VWN calculations are usually more accurate, in an absolute sense, than are the corresponding values obtained from the HF method. This is the case, even though they usually require less computational effort than HF calculations on the same molecule. It should be noted, however, that this does not necessarily mean that the VWN method provides a better representation of the trends in the bond dissociation energies calculated for a series of compounds (vide infra).

Electron correlation is accounted for in the MP4 method [33] by treating the difference between the actual coulombic repulsion between the electrons and the HF average as a perturbation. The MP4 energy is accurate through fourth order. By way of comparison, the HF energy corresponds to the sum of the zero and first order terms in the perturbation expansion of the energy. In contrast to the HF and LDA methods, which are based on single configuration wavefunctions, the MP4 energy includes contributions from many configurations and, as a result, it is almost always more accurate and precise. The coefficients in the MP4 multi-configuration wavefunction, however, are approximate in the sense that they do not diagonalize the molecular Hamiltonian. In fact, the wavefunction needs only to be accurate to second order to obtain fourth order accuracy in the energy.

The most accurate and computationally demanding of the calculations considered in the present study are based on the Gaussian - 2 (G2) approach [34]. This is more aptly described as a computational strategy because it consists of a series, rather than a single calculation. In the G2 approach, a hierarchy of relatively small perturbation and CI calculations are performed to account for the effects of the truncation of both the one- 


\section{Trends in Bond Dissociation Energies}

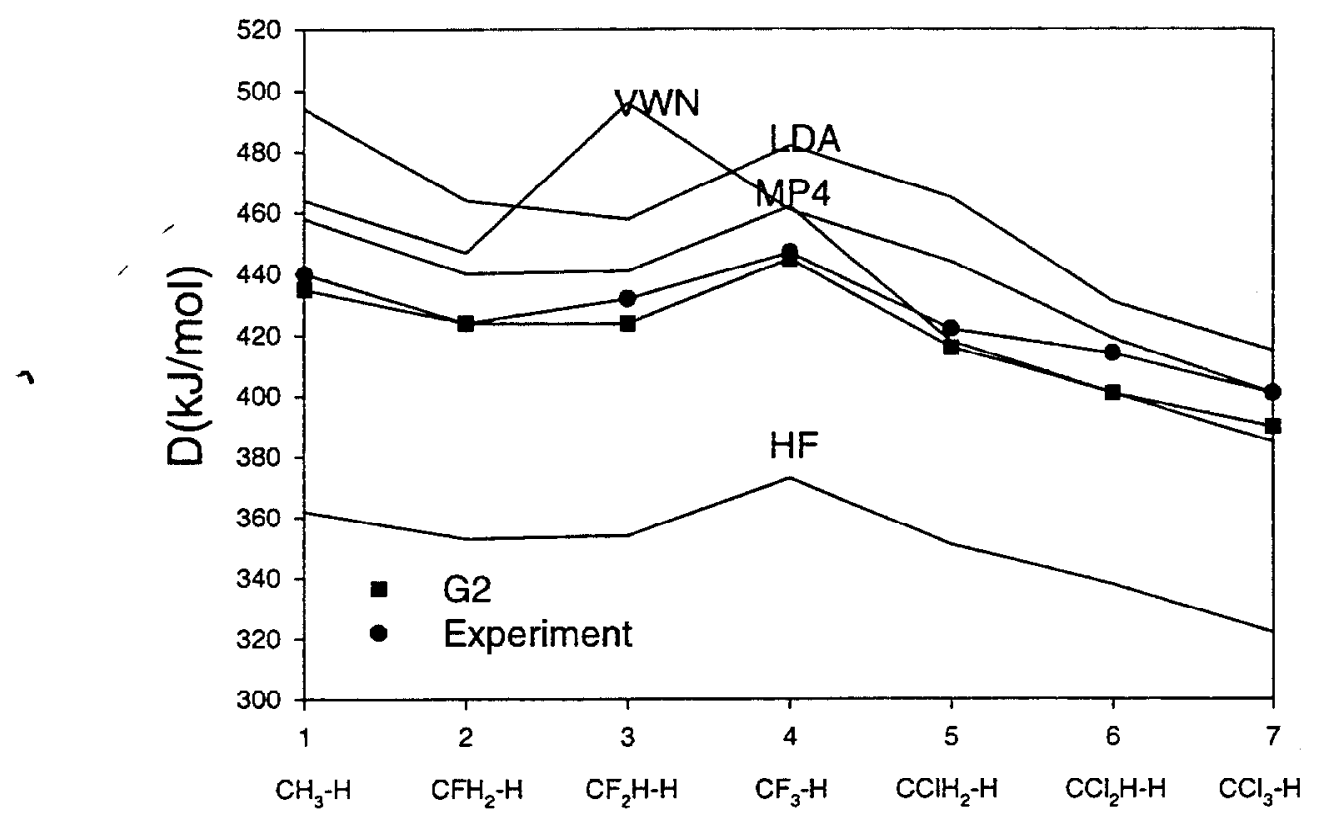

Molecule

Figure 2. A comparison of $\mathrm{C}-\mathrm{H}$ bond dissociation energies in the fluoro- and chloro-methanes obtained from the LDA, VWN, HF, MP4, and G2 (squares) methods with the experimental values (circles).

electron (orbital) and many-electron (configurational) basis sets, as opposed to a single large scale calculation; which would be prohibitive in all but the smallest molecules.

The bond dissociation energies calculated by each of the methods are plotted for the seven substituted methanes, which were the focus of this investigation, in Figure 2. All of the methods, with the exception of VWN, reproduce the trends in the experimental data. Only the G2 method, however, approaches quantitative agreement with the experimental values. The root mean square error of the $\mathrm{G} 2$ bond dissociation energies is about $3 \mathrm{~kJ} / \mathrm{mol}$, which corresponds to an uncertainty of almost $40 \%$ in the prediction of atmospheric lifetimes. While this is probably an acceptable level of accuracy for a screening method, bond additive corrections based on empirical data can be used to bring the computed bond energies into better agreement with experiment [35-38].

All of the methods, that were considered here in the context of the development of a screening method for environmental impact, are equally useful in evaluating bond dissociation energies and other parameters that are needed for the forcefields used in 
molecular mechanics, dynamics and Monte Carlo calculations. A description of a molecular dynamics model of thermal reactivity that makes use of a forcefield that has been modified to account for the major reactions involved in the thermal degradation of polymers is presented in the next section.

\subsection{Molecular Modeling of the Thermal Degradation of Polymers}

The research conducted in this laboratory has focused on the application of molecular modeling techniques to identify factors that affect the condensed phase thermal degradation chemistry of polymers in ways that result in a reduction in their flammability [5-9]. This effort has culminated in the development of a novel computer program, hereafter called MD_REACT, based on molecular dynamics. The feature that distinguishes MD_REACT from other MD codes is that it allows for the formation of new bonds from free radical fragments that are generated when bonds in the polymer break and, thereby, accounts for the chemical reactions that play a major role in the thermal degradation process (Figure 3) [39]. What follows is an overview of the theory and implementation of MD_REACT. This exposition is highlighted with results from recent applications performed as part of a general survey of fire retarded polymers. Included in this survey are blends of polypropylene with silica gel, three representative bromine-containing additives, and a polypropylene/graphite layered nanocomposite. Some preliminary results from a model validation study of the rate of beta-scission reactions in a small gas phase molecule (n-pentane) are also presented.

The motivation behind the development of MD_REACT was to create a versatile model that could be used to study thermal degradation at a molecular level in a wide range of polymers. The strategy employed to accomplish this objective was to interface our program for performing reactive dynamics on simple vinyl polymers [6] with Discover 95 , a commercially available molecular dynamics code offered by Molecular Simulations, Inc. (MSI)* [40].

The interface between the programs is established using an inter-process communication protocol (IPC) to pass coordinates, forces and connectivity information between MD_REACT, which computes the reactive forcefield, and Discover 95, which updates the molecular structure on the basis of the solution to the equations of motion.

* Certain commercial equipment, instruments, materials, or companies are identified in this paper in order to adequately specify the experimental procedure. This in no way implies endorsement or recommendation by NIST. 


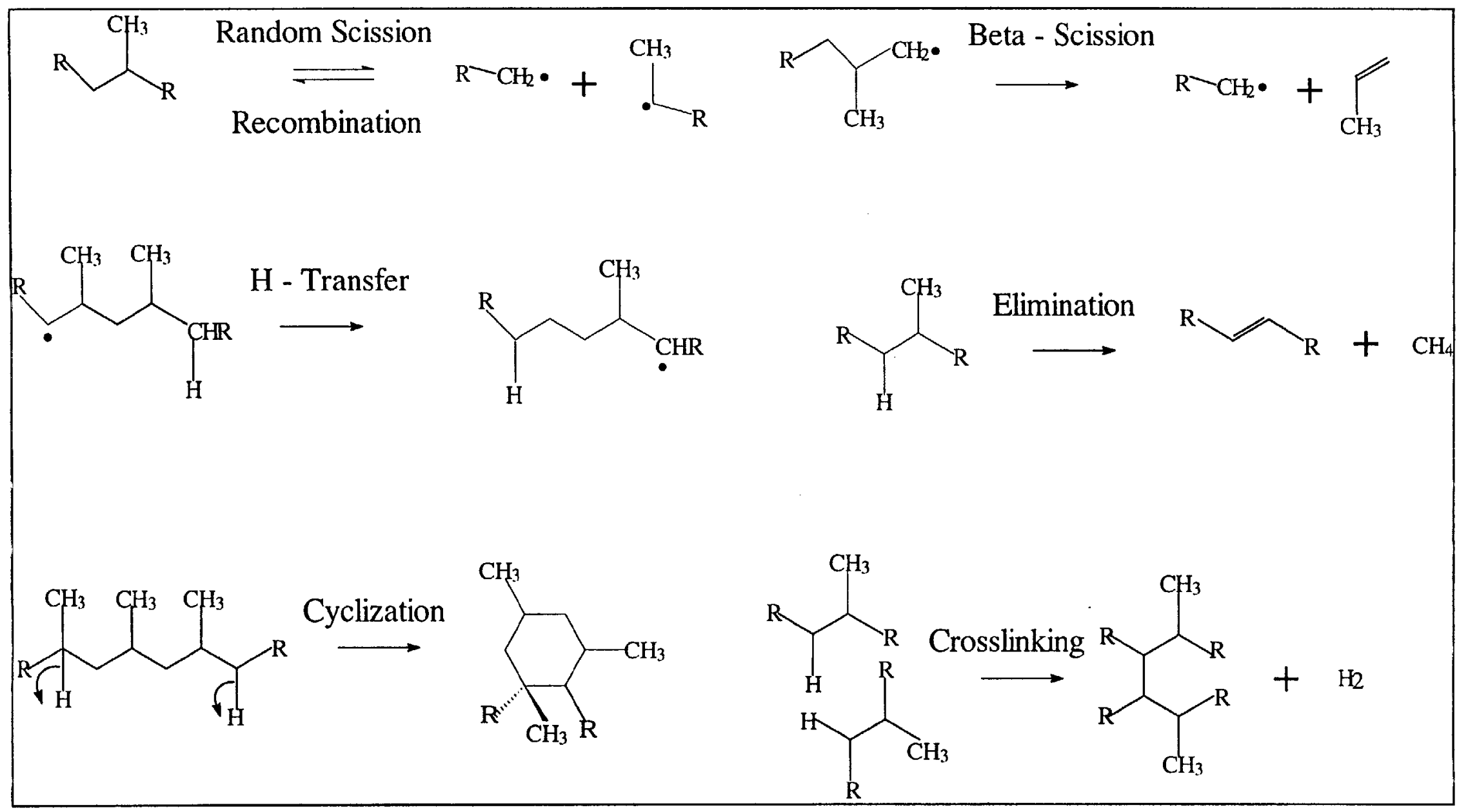

Figure 3. Major reaction pathways in the thermal degradation of polymers. 
The input file for the Discover 95 run contains a command string that initializes an IPC connection [41] in server mode and launches MD_REACT as an external process. A series of database operations that identify and replace the high-energy bonds are executed at every time step.

The basis of MD_REACT is molecular dynamics. This technique consists of solving Hamilton's equations of motion (Eq. ( 8 )) for each of the $3 \mathrm{~N}$ molecular degrees of freedom. The Hamiltonian of the model polymers considered in the present investigation was derived from the Centralized Valence Forcefield (CVFF) summarized in Eq. (10). What follows is an explanation of the individual terms that contribute to this forcefield description of the potential energy of a molecule.

The energy required to stretch the covalent bonds is represented by a Morse potential, which is given by Eq.( 13 ).

$$
V_{b}=D\left[1-\exp \left(-\alpha\left(r-r_{e}\right)\right)\right]^{2}-D
$$

In this equation, $r$ is the distance between bonded atoms $i$ and $j, r_{e}$ is the equilibrium bond length, and $\alpha=\left[\mathrm{k}_{\mathrm{b}} /(2 \mathrm{D})\right]^{1 / 2}$, where

$$
k_{b}=\left(\frac{\partial^{2} V_{b}}{\partial r^{2}}\right)_{r=r_{c}}
$$

is the force constant. The bond dissociation energies (D) have, in many cases, been altered from their original CVFF values in an attempt provide a better description of the thermal decomposition reactions summarized in Figure 3 . The new values were obtained from a variety of methods including experimental data and G2 calculations performed on monomers and other model compounds with representative bonds.

The potential energy for bond bending is represented by

$$
V_{a}=S(i j) S(j k) k_{\theta}\left(\theta-\theta_{e}\right)^{2},
$$

where $\theta$ denotes the angle determined from the dot product of the normalized bond vectors between three adjacent atoms $(\mathrm{i}, \mathrm{j}$ and $\mathrm{k})$ and the $\mathrm{S}(\mathrm{ij})$ and $S(\mathrm{jk})$ are switching functions, which are defined below. The force constant, $\mathrm{k}_{\theta}$, is defined, in analogous fashion to $k_{b}$, as the second derivative of the potential energy evaluated at the equilibrium bond angle $\left(\theta_{\mathrm{e}}\right)$.

Rotations about covalent bonds are restricted by the torsional potential

$$
V_{t}=S(i j) S(j k) S(k l) k_{\phi}\left[1+\cos \left(n \phi-\phi_{e}\right)\right] \text {. }
$$

The dihedral angle, $\phi$, is defined by the three bond vectors between four adjacent atoms (i,j,k and $\mathrm{l}$ ). The parameters, $\mathrm{k}_{\phi}, \mathrm{n}$, and $\phi_{\mathrm{e}}$ determine the height, multiplicity, and position of the barrier to internal rotation.

The nonbond potential energy consists of two terms corresponding to the Van der Waals and electrostatic interactions. These are represented by 


$$
V_{n b}=\varepsilon\left[\left(\frac{r^{*}}{r}\right)^{2}-2\left(\frac{r^{*}}{r}\right)^{6}\right]+\frac{\delta_{i} \delta_{j}}{r},
$$

where $\delta_{i}$ and $\delta_{j}$ are the partial charges on nonbonded atoms separated by a distance, $r$. The parameters $r^{*}$ and $\varepsilon$ in the Van der Waals term determine the position of the minimum and the depth of the potential well, respectively.

The switching functions, S(ij) in Eqs.( 15 ) and ( 16 ) are used to turn the bending and twisting forces on and off as the corresponding bonds are formed and broken. It is the presence of these terms that distinguishes the reactive forcefield used in MD_REACT from the CVFF and other conventional forcefields. The switching functions used in previous versions of MD_REACT were based on the hyperbolic tangent function in Eq.( 18 ).

$$
S(i j)=\frac{I}{2}\left(I-\tanh \left(a\left(r_{i j}-r_{d}\right)\right)\right)
$$

The justification for using this functional form is that it has the character of a step function, while preserving continuity of the derivatives that are needed for the evaluation of the forces. The parameters $\mathrm{a}=40 \mathrm{~nm}^{-1}$ and $\mathrm{r}_{\mathrm{d}}$, which is the inter-atomic distance at which the energy in the bond is within $\mathrm{k}_{\mathrm{B}} \mathrm{T}$ of dissociation, determine the sharpness and the position of the transition from $S=1$ to $S=0$, respectively. In the most recent version of MD_REACT, fractional bond orders, as defined in Eq.( 19 ), are used to switch the forces.

$$
S(i j)=\left\{\begin{array}{cc}
1 & r \leq r_{e} \\
\frac{-V_{b}(i j)}{D(i j)} & r>r_{e}
\end{array}\right.
$$

This formulation facilitates the description of hypervalent transistion states for hydrogen transfer and similar reactions that play a major role in the thermal degradation process. The energy and forces calculated in Discover 95 do not include the contributions due to the presence of these switching functions. These corrections are computed in MD_REACT and passed to Discover 95 in the form of a restraint on the energies and forces [41].

Bond dissociation and formation are simulated in MD_REACT by the following algorithm. A list of the free radical sites, that are generated when bonds in the polymer break, is updated at every time step. The criterion used in this program is that the atoms participating in a covalent bond become free radicals when $\mathrm{V}_{\mathrm{b}}$ comes within $n \mathrm{k}_{\mathrm{B}} \mathrm{T}$ of the dissociation energy, where $n$ is an integer specified at the onset of the simulation. Although a large value for $n$ ensures that there will be a lot of free radicals, they are inconsequential unless a lower energy pathway is found (vide infra). The program generates a list of potential bonds consisting of all possible covalent interactions between the available free radicals and retains those corresponding to the lowest energy subject to 
the constraints imposed by the atomic valence. This is accomplished by invoking a section of code that sorts the potential bonds in order of increasing energy and accepts new bonds until the valence of the participating atoms is filled. Of course, the number of bonds to an atom can, and frequently does, decrease from its maximum value as a result of bond scission reactions. The bond table in Discover 95 is modified and the trajectory of the polymer is updated accordingly.

In order to extract meaningful information from the simulations of thermal degradation, the disparity between the time scales associated with laboratory measurements, such as the cone calorimeter (seconds) and those accessible to atomistic simulations (picoseconds) must be overcome. Our approach to this problem is to make use of a simple kinetic model to identify the reactions which determine the global rate of mass-loss and the distribution of products. The following expression [42]:

$$
\frac{1}{m(t)} \frac{d m(t)}{d t}=-\left(2 k_{i}(T)+[R] k_{I}(T)\right) Z(T)
$$

is consistent with the results obtained from our simulations of the thermal degradation of polypropylene. In this equation, $\mathrm{m}(\mathrm{t})$ denotes the mass of the residual polymer at time, $t$,

$$
k_{i}(T)=\omega \exp \left(-\frac{E_{a}}{k_{B} T}\right)
$$

is the rate constant for the random scission initiation reaction, and $\mathrm{k}_{\mathrm{I}}(\mathrm{T})$ is the rate constant for chain transfer scission. In the latter case, a $\beta$-scission occurs on a polymer after it transfers a hydrogen atom to a free radical polymer fragment (frpf). The steadystate concentration of frpfs, [R], which is assumed to be constant throughout the data collection process, is given by Eq. ( 22 )

$$
[R]=\frac{2 k_{i}(T) d_{0}}{k_{i}(T) m_{0}},
$$

where $k_{r}(T)$ is the rate constant for termination, $d_{0}$ is the density of the polymer sample and $\mathrm{m}_{0}$ is the molecular weight of the repeat unit. The zip-length,

$$
Z(T)=\frac{k_{p}(T)}{\left(k_{t}(T)+k_{t}(T) \frac{d_{0}}{m_{0}}\right)},
$$

is the number of volatile fragments (including, but not restricted to, monomers) produced before the frpf is terminated (i.e. converted back to a stable, nonvolatile molecule) either by the transfer of a hydrogen from a neighboring polymer chain or by the dissociation of a hydrogen or methyl group from the $\beta$-bond.

The disparity in the time scales corresponding to the initiation reactions, which have activation barriers on the order of $320 \mathrm{~kJ} / \mathrm{mol}$ or more, and the propagation and termination reactions, which have activation barriers of $160 \mathrm{~kJ} / \mathrm{mol}$ or less, is resolved by performing independent simulations at temperatures appropriate for each of these processes. Thus, a typical computer experiment involves at least two sets of simulations. 
An artificially high temperature (typically, $>2000 \mathrm{~K}$ ) is used in the first series of simulations for the purpose of generating a sufficient population of frpfs to initiate the propagation/termination reactions, which constitute the second stage of the computer experiment. These simulations can be performed at lower temperatures because of the relatively small activation energies associated with $\beta$-scission, which is the dominant mechanism in the propagation and first order termination reactions. The global rate of mass-loss at thermal degradation temperatures, $T_{d}$, may then be obtained from Eq. ( 20 ) by extrapolation of the temperature dependent rate constants computed using data collected in this sequence of MD simulations. Work on the incorporation of a third stage, that will simulate the transport of thermal degradation products through the polymer melt, is in progress.

Periodic boundary conditions are implemented by enclosing the model polymer in a unit cell with dimensions that are consistent with the experimental density. The bulk system is simulated by accounting for interactions between the atoms in the polymer and ghost atoms that are created by replicating the unit cell in space (see Figure 10). This makes it possible to maintain realistic densities during simulations of the thermal degradation of isolated chains. The use of periodic boundary conditions also facilitates the assessment of thermal stability, since the rate of mass loss from the degrading polymer can be computed by tabulating the mass of the fragments diffusing outside of the central unit cell as a function of time.

\section{2a Pentyl Radical}

The reactions that are responsible for most of the mass loss during the thermal degradation of polymers are hydrogen transfer and beta-scission. Obviously, the credibility of our model for predicting the thermal stability and flammability of polymers would be questionable if it failed to provide reasonable estimates for the rate constants of these reactions. Here, we examine the predictive capability of the model by comparing the computed and experimental rate constants for beta-scission in n-pentyl radical for a series of temperatures between $1000 \mathrm{~K}$ and $1600 \mathrm{~K}$. The conformations of the pentyl radical at the onset of a representative simulation and just after a beta-scission are compared in Figure 4.

The computer simulations were performed as single molecule MD calculations (i.e.,without periodic boundary conditions) at a constant energy until the reaction occurred. The unimolecular rate constants were computed as $t_{r \times n}{ }^{-1}$, where $t_{r \times n}$ is the mean reaction time corresponding to three independent realizations of the initial momentum 

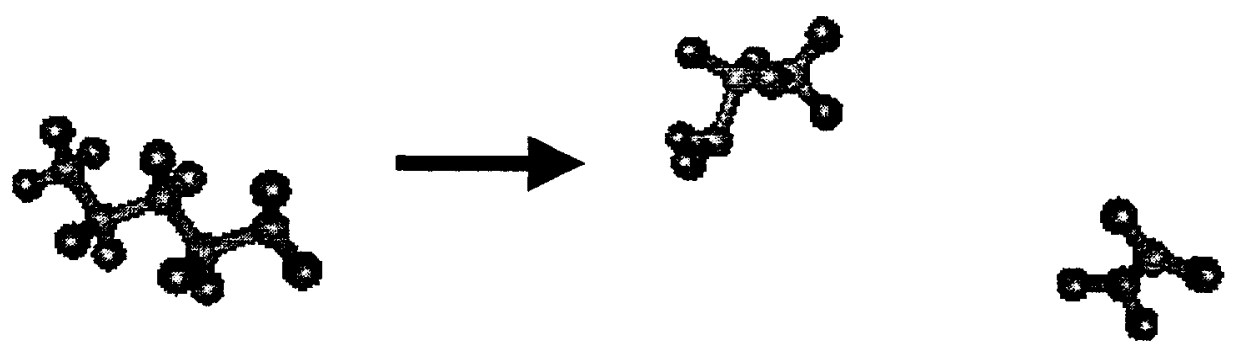

Figure 4. Still frames taken at the beginning (left) and end (right) of a simulation of the thermal degradation of n-pentyl radical.

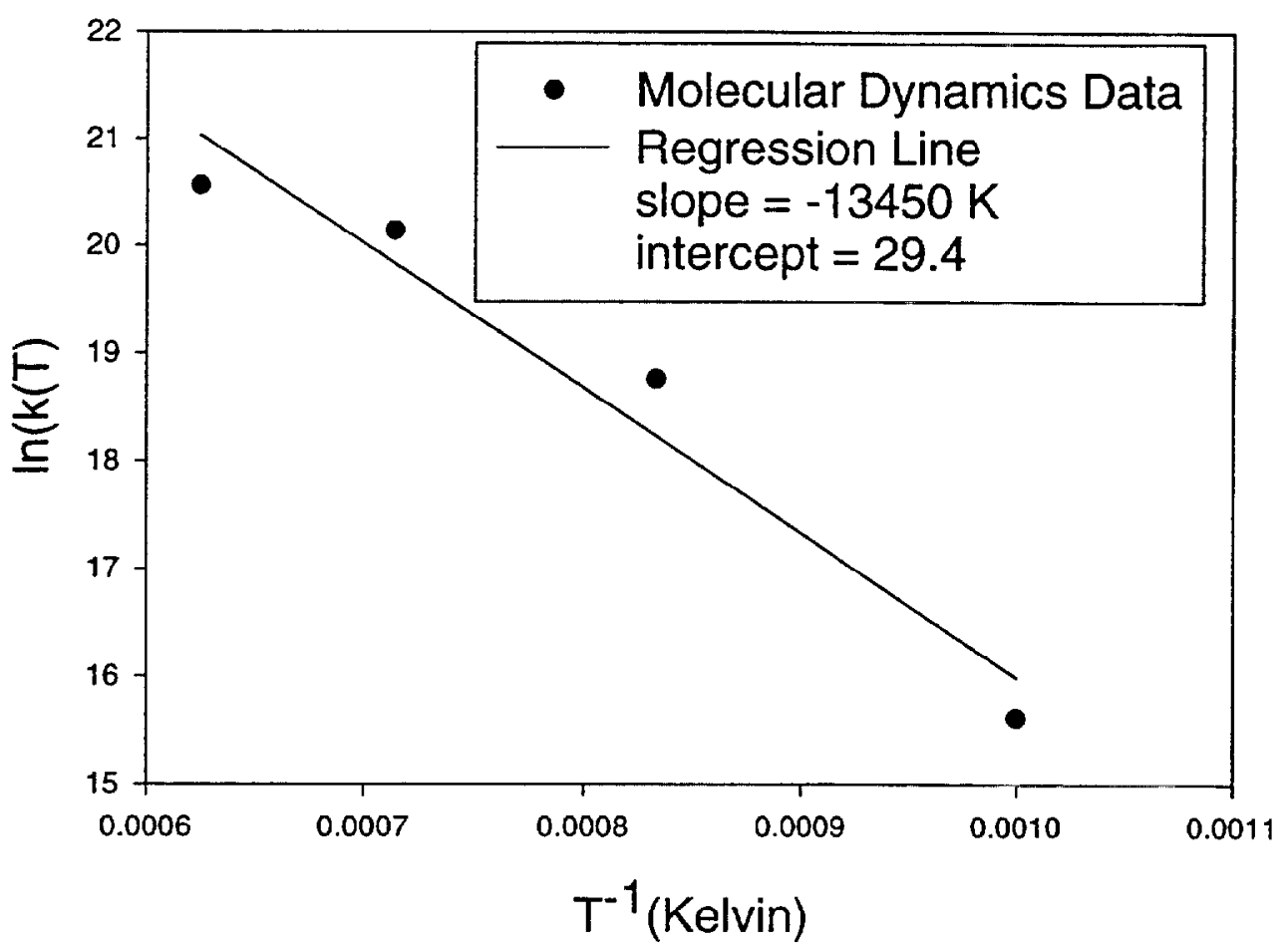

Figure 5. Ahrrenius plot of the rate constants for $\beta$-scission of the first $C$ - $C$ bond in n-pentyl radical. 
distribution, at each of the specified excitation energies. These results are listed in Table II. The uncertainties were estimated as the standard deviations from the mean

Table II. Mean Reaction Times from Simulations of Beta-Scission in n-Pentyl Radical

\begin{tabular}{cc}
\hline Energy $(\mathrm{kJ} / \mathrm{mol})^{\mathrm{a}}$ & $\mathrm{t}\left(\mathrm{\times} \mathrm{10^{12 }} \mathrm{s}\right)^{\mathrm{b}}$ \\
\hline $502 \pm 4$ & $1733 \pm 595$ \\
$544 \pm 4$ & $533 \pm 120$ \\
$573 \pm 4$ & $421 \pm 136$ \\
$606 \pm 4$ & $387 \pm 106$ \\
\hline
\end{tabular}

a. Computed as the mean of the time averaged energy from 3 simulations.

b. The average length of time before a reaction occurred in 3 simulations.

reaction time. The total simulation time spanned by these calculations was about $9 \times 10^{-9}$ $\mathrm{s}$, which corresponds to almost $10^{5}$ vibrations of the critical bond; each of which may be viewed as an independent bond dissociation experiment.

The temperature dependent rate constants were computed by numerical integration of Eq. (24),

$$
k_{\infty}(T)=\int_{E_{0}}^{\infty} P(E, T) k_{u n i}(E) d E,
$$

where $\mathrm{k}_{\mathrm{uni}}(\mathrm{E})=\mathrm{t}_{\mathrm{rxn}}{ }^{-1}$ is the unimolecular rate constant at the specified energy listed in Table II and

$$
P(E, T)=\frac{E^{S-1} \exp \left(-\frac{E}{k_{B} T}\right)}{\Gamma(S)\left(k_{B} T\right)^{S}}
$$

is the classical probability that a molecule in equilibrium with a reservoir at the temperature, $T$, has an energy, $E$ [43]. In this equation, $S=3 N-6$ is the number of vibrational degrees of freedom in the molecule, $T$ is the absolute temperature, and $\Gamma(S)$ is the gamma function which is equal to (S-1)!. The results obtained from the integration of Eq. ( 24 ) are listed in Table III. The uncertainties were estimated by propagating the standard deviations from the mean reaction time in the integral. The large variability in these values reflects the statistical nature of the thermal degradation process. The kinetic constants corresponding to these rate constants, which were obtained from the Ahrrenius plot in Figure 5 are $E_{a}=112 \pm 17 \mathrm{~kJ} / \mathrm{mol}$ and $A=0.6 \times 10^{13} \mathrm{~s}^{-1}$. 
Table III. Comparison between Computed and Experimental Rate Constants

\begin{tabular}{ccc}
\hline $\mathrm{T}(\mathrm{K})$ & $\mathrm{k}_{\infty}\left(\mathrm{x} 10^{-8} \mathrm{~s}^{-1}\right)^{\mathrm{a}}$ & $\mathrm{k}_{\infty}\left(\times 10^{-8} \mathrm{~s}^{-1}\right)^{\mathrm{b}}$ \\
\hline 1000 & $0.061 \pm 0.027$ & 0.063 \\
1200 & $1.4 \pm 0.55$ & 0.68 \\
1400 & $5.6 \pm 2.4$ & 3.7 \\
1600 & $8.5 \pm 3.4$ & 13 \\
\hline
\end{tabular}

a. From Eq.( 24 ).

b. From Eq. ( 26 ).

The familiar Ahrrenius expression given in Eq.( 26 ) is known to provide an accurate description of the kinetics of unimolecular reactions in the high pressure limit when the rate of deactivation by collisions with other molecules is much greater than the rate of unimolecular decomposition. Thus, the experimental rate constants in this regime can be obtained from

$$
k_{\infty}(T)=\omega \exp \left(\frac{-E_{0}}{L k_{B} T}\right),
$$

where $\omega$ is the frequency of energy exchange between the vibrational modes, $L$ is Avogadro's number and $E_{0}$ is the minimum or critical energy required for the unimolecular reaction. The kinetic parameters determined from measurements made in a single pulse shock tube at the high pressure limit over the temperature range from 850 $1000 \mathrm{~K}$ are: $\omega=1.0 \times 10^{13} \mathrm{~s}^{-1}$ and $\mathrm{E}_{0}=118.7 \mathrm{~kJ} / \mathrm{mol}$ [44]. Substitution of these values into Eq. 26 ) gives the experimental rate constants listed in Table III. Although the rate constants obtained from the MD simulations are in reasonable agreement, the fact that the discrepancy exceeds the uncertainty of the calculations at some temperatures suggests that there may be additional sources of error. Included in the list of possibilities is the use of classical dynamics to simulate chemical reactions that, in reality, are quantum events.

\section{2b Silica Gel}

Results obtained from experimental studies conducted in this laboratory have indicated that the flammability of polypropylene and other polymers is reduced by the addition of small quantities of silica gel ( $\sim 10 \%$ of the total mass) [45]. The effect of silica gel on the thermal degradation of polypropylene was investigated by comparing the results of simulations in the presence and absence of this additive.

The model polymer consisted of 4 chains of isotactic polypropylene, each containing 48 propylene monomers (Figure 6). Two models of the silica gel, one with 81 $\mathrm{SiO}_{4}$ moieties and a maximum pore diameter of $10 \AA$ and the other with $215 \mathrm{SiO}_{4}$ moieties and a maximum pore diameter of $30 \AA$, were examined in an effort to determine the effect of pore diameter on the thermal degradation of the polymer. The initial geometries for the polymer/silica systems were obtained by minimizing the CVFF 
energies until the maximum derivative was less than $4.2 \mathrm{~J} /(\mathrm{mol} \AA)$ using the Polak Ribiere conjugate gradient algorithm in Discover 95 [41].

The simulations were performed with periodic boundary conditions in two steps. First, a population of free radical polymer fragments was generated by integrating the First, a population of free radical polymer fragments was generated steps) using the full CVFF forcefield with cross terms coupling adjacent bonds, angles

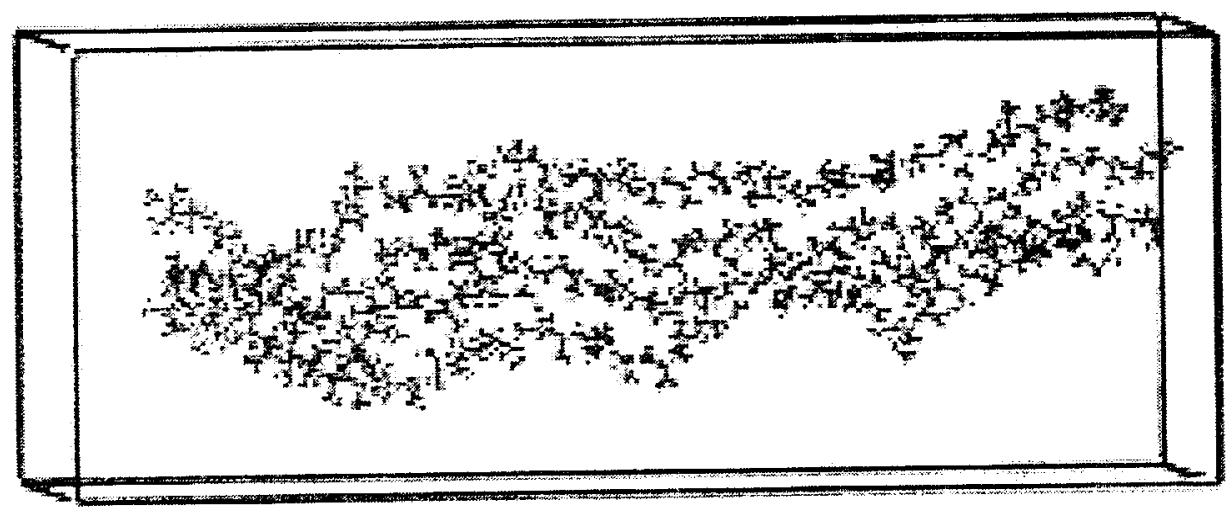

Figure 6. The model polymer consisting of 4 chains each comprised of 48 propylene monomers.

and torsions. The purpose of including cross terms was to facilitate an efficient transfer of thermal energy into the stretching modes that are responsible for bond dissociation. The reactive dynamics were then carried out at a constant temperature of $873 \mathrm{~K}$ for $5.0 \times 10^{-12}$ $\mathrm{s}$ (5000 time steps) using the reactive forcefield described above. Only the atoms in the polypropylene were dynamic. The positions and connectivities of the $\mathrm{Si}, \mathrm{O}$ and $\mathrm{H}$ atoms in the silica were fixed throughout the simulations. This was done to make the simulations more computationally tractable. Benchmark studies, where these atoms were free to move, indicated that the deformations from the initial structures were minor and that the strategy of constraining the movement of the silica was, therefore, a realistic approximation. 
Cone calorimeter measurements on polypropylene/silica gel blends have indicated that pore diameter has a significant effect on the rate-of-heat release of the burning polymer [45]. In fact, the range of pore diameters spanned by our models is about an order of magnitude smaller than the range manifested in the silica gels used in the experiments. The molecular weight of the model polymers is likewise about an order of magnitude smaller than the average value of $\mathrm{Mn}$ attributed to the actual polymers. Thus, in using the model calculations to interpret the experimental results, we are assuming that the observed effects depend only on the relative, and not on the absolute, size of the molecules involved.

The effect of the silica gel was examined by comparing simulations where the nonbond interactions (electrostatic and Van der Waals forces) between the silica and the polymer were turned on (representing a blend of polymer and silica) to identical simulations where these interactions were turned off (representing a non-interacting mixture of polymer and silica with the same density as the blend). The corresponding mass loss rates, determined by numerical differentiation of the mass of polymer degradation fragments diffusing out of the unit cell as a function of time, are compared in Figure 7. Negative values, which result when fragments diffuse back into the cell, were observed in some simulations. Presumably, these negative contributions cancel when the rates of mass loss are averaged over macroscopic scales of time and distance. The uncertainties in the rates, estimated from the average standard deviation of the differences in the rate of mass loss obtained from independent simulations performed at the same temperature, are 0.07 and 0.05 (in units of fractional mass loss per $10^{-12} \mathrm{~s}$ ) for the pure polymer and the blend, respectively. The interactions between the polymer and silica appear to have a stabilizing effect that would be expected to result in a reduction in flammability. A detailed review of the computer animations of the corresponding trajectories suggests further that the fragments from the degrading polymers tend to get trapped in the pores of the silica. This effect is apparent in Figures 8 and 9, where the last frames from simulations of the thermal degradation of polypropylene in the presence (right) and absence (i.e., when there are no interactions between the polymer and its degradation products with the silica) of the silica gel are compared for the small and large pore silica, respectively. In general, the large pore silica appears to be more effective because it can trap a greater mass of polymer degradation products than the small pore silica.

\section{2c Nanocomposites}

Polymer layered nanocomposites, which are characterized by the molecular level incorporation of an inorganic additive (commonly alumino silicate clays) into an organic polymer, can have unique properties compared to conventional filled polymer systems $[46,47]$. Research conducted by Gilman et al. has demonstrated that some nanocomposites of polymer and clay exhibit a significant reduction in flammability, as compared to immiscible mixtures of the same components, even at compositions which contain as little as $2 \%-5 \%$ clay $[48,49]$. Simulated thermal degradation experiments were performed to 


\section{Rate of Mass Loss}

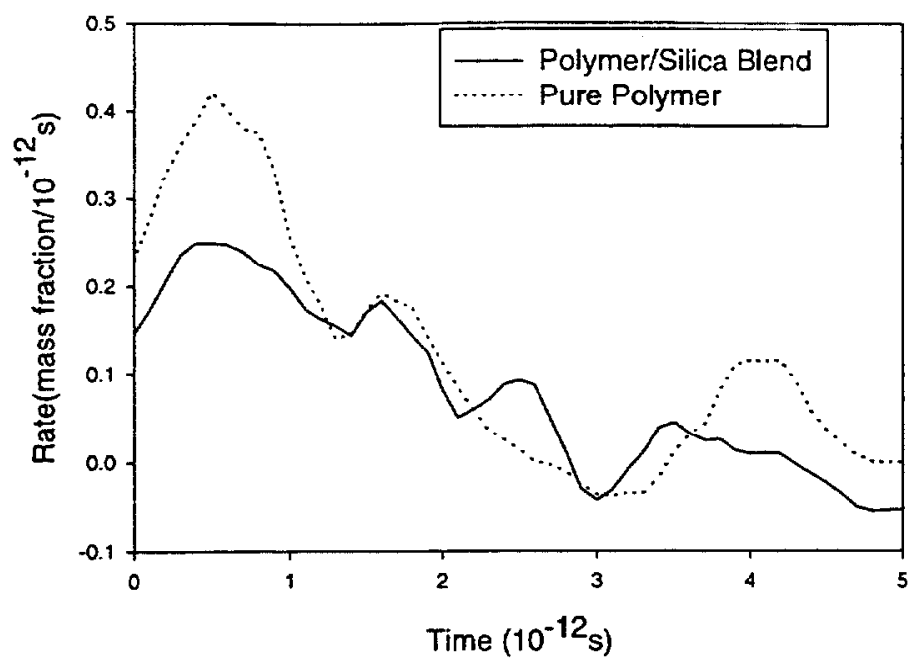

Figure 7. The rates of mass loss from the polymer/silica blend are compared to the corresponding values obtained for the non-interacting mixture of the same components.

investigate the source of this dramatic increase in fire resistance at a molecular level.

The model of the polymer nanocomposite consisted of the 4 polypropylene chains used in the previous study situated above a graphite sheet constructed from about 600 carbon and 80 hydrogen atoms that were used to terminate the edges. A series of nanocomposite structures with the polymer intercalated between graphite layers separated by a variable distance, $b$, was obtained by annealing the model polymer and graphite inside of a unit cell with the following dimensions: $a=10.0, c=3.0$ and $b=(2.5,2.8$, 3.0, 3.2 and 5.0) $\mathrm{nm}$. The same models of the polymer and graphite were used in all of the structures. That is, only the distance between the graphite sheets and, consequently, the density of the composite was allowed to change from one simulation to the next. The $b=$ 3.0 nanocomposite is displayed in Figure 10.

The simulated annealing was performed as follows. The polymer/graphite assembly was heated at $500 \mathrm{~K}$ for 100 time steps of molecular dynamics and then allowed to relax during 100 iterations of the Polak Ribiere conjugate gradient minimization. After 10 cycles, an optimized conformation was obtained by minimizing the full CVFF energy until the maximum derivative was less than $4.2 \mathrm{~J} /(\mathrm{mol} \AA)$. This process was repeated until the energy of the last optimized conformation was the lowest value attained during the course of the simulated annealing procedure.

The molecular dynamic simulations were performed using periodic boundary conditions in two stages. In the first stage, a population of free radical polymer fragments was generated by integrating the equations of motion over a constant temperature path of $873 \mathrm{~K}$ for $0.05 \times 10^{-12} \mathrm{~s}$ using the full CVFF force field with cross terms coupling adjacent bonds, angles, and torsions. The bond stretching energies and forces were scaled by a factor of 0.2 to ensure that there was a sufficient population of free radical fragments 

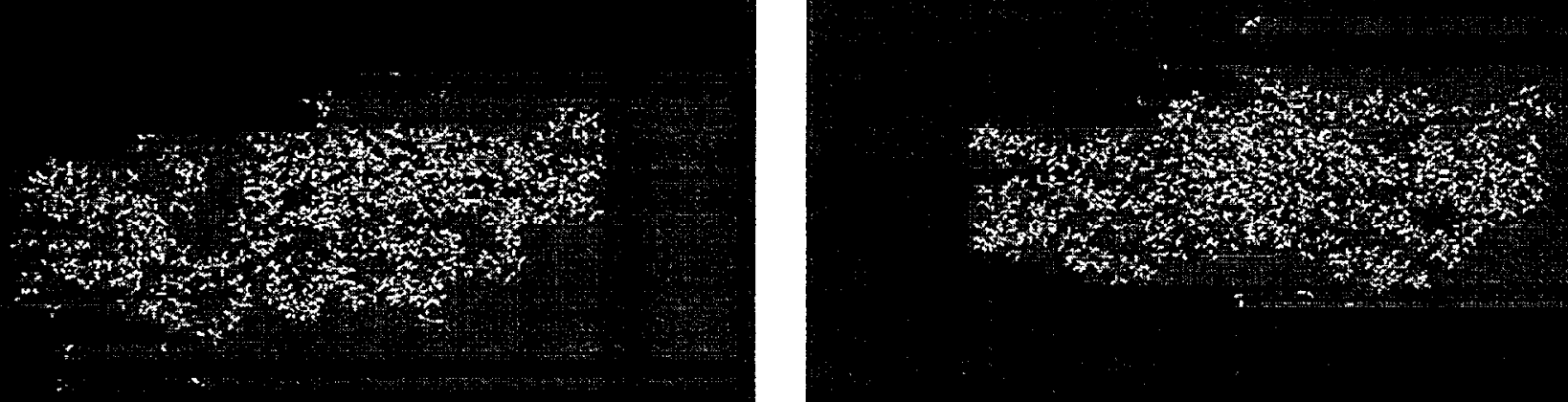

Figure 8. Thermal degradation of the non-interacting mixture (left) and the blend with the small pore silica (right). The animated trajectories indicate that the fragments from the degrading polymers in the blend tend to get trapped in the pores of the silica.
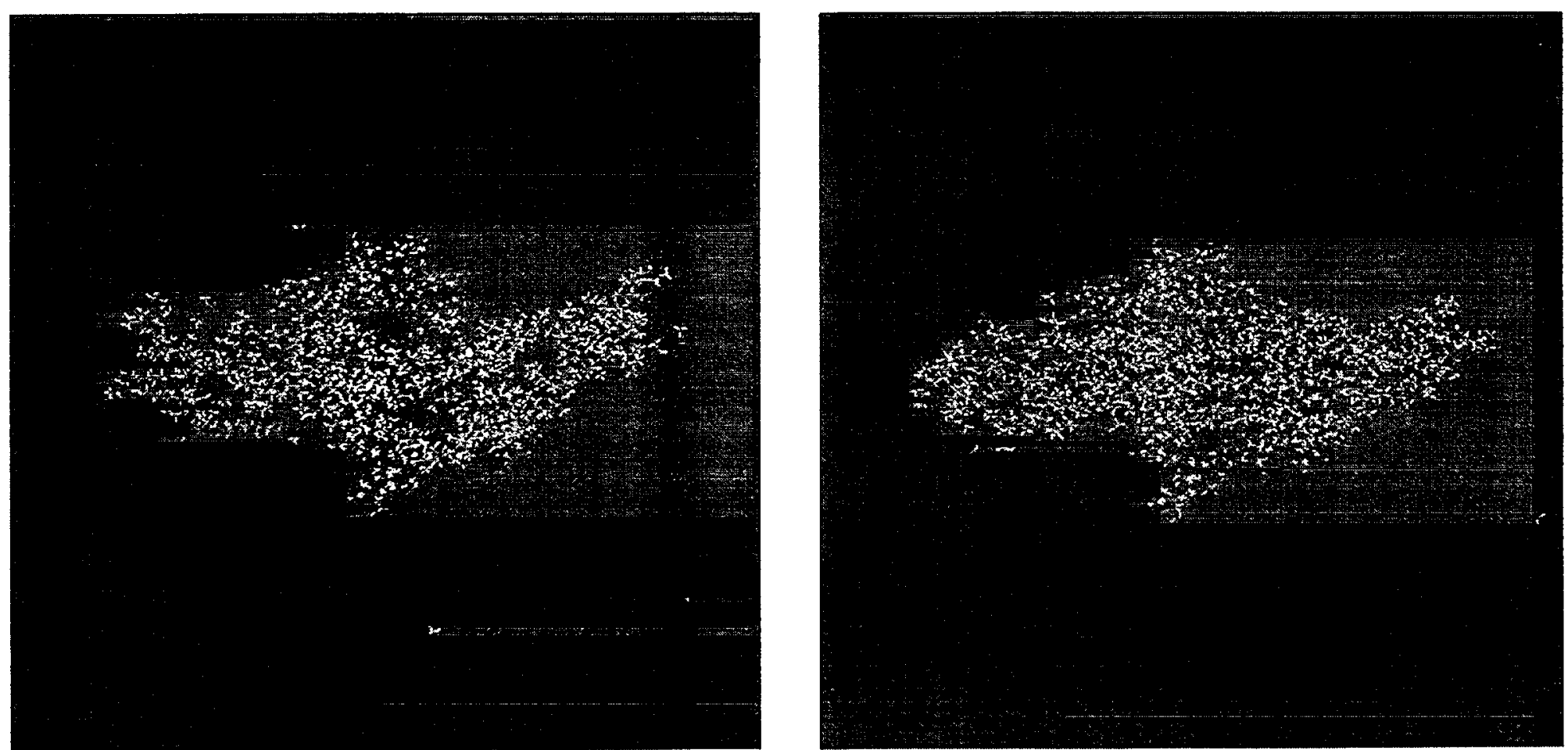

Figure 9. Thermal degradation of the non-interacting mixture (left) and the blend with the large pore silica (right). The large pore silica appears to be morc cffective than the small pore silica because it can trap a greater mass of polymer degradation products. 
to initiate the propagation and termination reactions (i.e.,depolymerization, hydrogen transfer, chain stripping, cyclization, crosslinking and radical recombination). The bond bond scale factor was then set back to unity at the onset of the second stage where the propagation/termination reactions were simulated. This stage of the simulation was carried out using the reactive forcefield described above at $873 \mathrm{~K}$ for $5.0 \times 10^{-12} \mathrm{~s}$. The positions and connectivities of the carbon and hydrogen atoms in the graphite were fixed throughout the simulations.

The relative thermal stabilities of the polypropylene/graphite nanocomposites were assessed by comparing the average rates of mass loss for each system during the second (reactive) stage of the simulations. Each value was obtained as the arithmetic mean of a set of 51 instantaneous values (giving a time averaged rate), which in turn, were (ensemble) averaged over 3 independent simulations corresponding to different realizations of the initial momentum distribution for the specified temperature. The uncertainties, which are represented by error bars, are the standard deviations of the time averaged rates from these 3 simulations.

The average rates of mass loss, from the nanocomposites and non-interacting mixtures of the polymer and graphite with the same densities (obtained by turning off the nonbonding interactions between the polymer and the graphite), are plotted as a function of the distance of separation between the graphite layers in Figure 11. The reduction in the average rates of mass loss (with respect to the non-interacting mixtures) that was observed in simulations of the thermal degradation of the $(2.8,3.0$ and 3.2) $\mathrm{nm}$ nanocomposites is consistent with the results obtained from radiative gasification measurements made on intercalated polypropylene and polystyrene nanocomposites [49]. The stabilization of the polymer is most pronounced in the $b=3.0 \mathrm{~nm}$ nanocomposite and approaches zero at $b=5.0 \mathrm{~nm}$, when the graphite layers are too far apart for there to be $a$ significant interaction between them. At these large distances of separation, the interactions are almost exclusively between the polymer and the graphite. This should approximate what occurs in the delaminated nanocomposites where the graphite layers are individually dispersed in the polymer matrix. At $b=2.5 \mathrm{~nm}$, the nanocomposite is actually destabilized. This is a consequence of the repulsive nature of the nonbonding forces which predominate when the polymer chains are crowded into a small volume. In fact, this effect is so pervasive that we could not even locate a stable conformation for a layered nanocomposite structure with $b$ less than $2.5 \mathrm{~nm}$ using the simulated annealing procedure described above. In retrospect, this could have been anticipated because these nanocomposites are known to form delaminated, rather than layered structures, when the mass fraction of polymer exceeds a critical value that corresponds to about $80 \%$ in the nylon-6/clay nanocomposites [47].

The nature of the stabilization effect observed for the $(2.8,3.0$ and 3.2$) \mathrm{nm}$ nanocomposites is revealed by comparing the computer animations of the trajectories corresponding to the nanocomposites and the non-interacting mixtures. In general, the polymers in the $b=(2.8,3.0$ and 3.2$) \mathrm{nm}$ nanocomposites lost fewer fragments and 


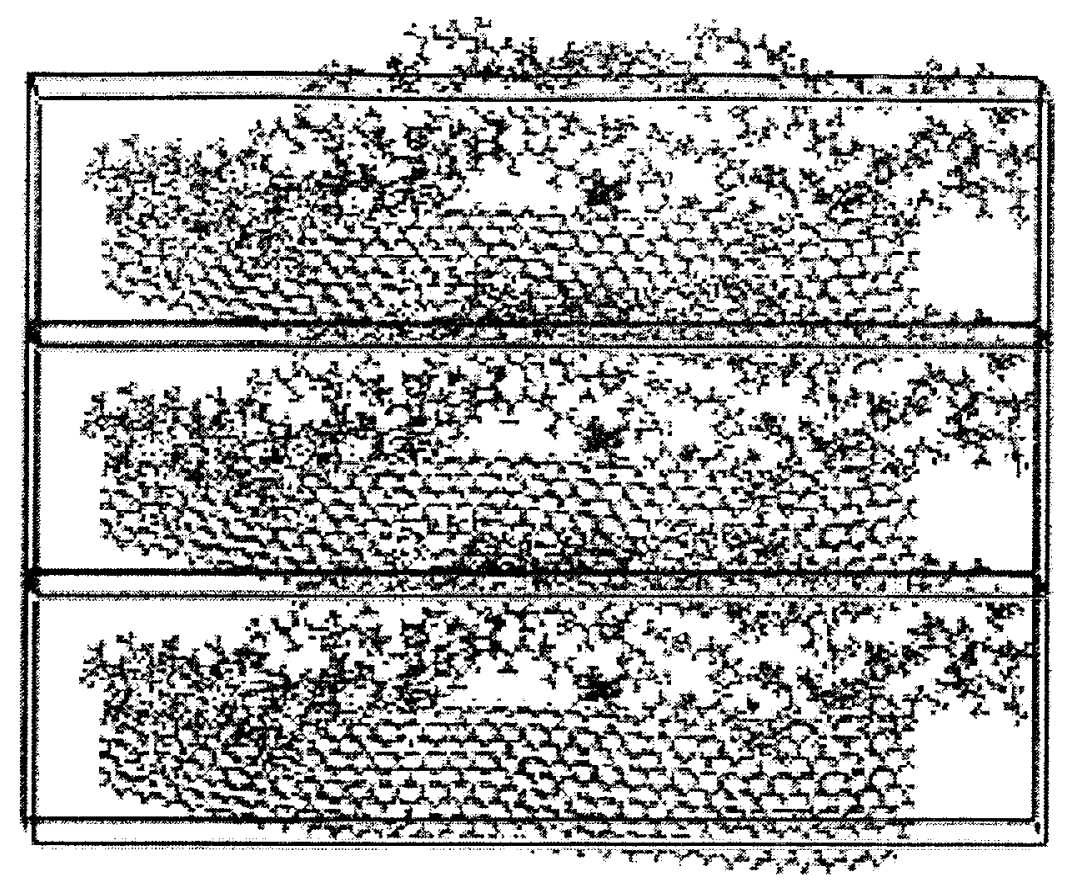

Figure 10. Periodic display of the model polypropylene/graphite nanocomposite.

retained their shape longer than the polymers in the non-interacting mixtures with the same densities. The most obvious difference was that the fragments generated by the

degradation of the model polymer in the nanocomposites had a pronounced tendency to collide with the graphite and bounce back into the central unit cell where they could undergo recombination reactions with other free radical polymer fragments, rather than escape from the melt as combustible fuel.

The observation that the thermal stability of the polymer increases when it is intercalated but is not affected by the presence of the graphite when the layers delaminate is consistent with thermogravimetric data that indicate that intercalated nanocomposites are more thermally stable than delaminated nanocomposites $[48,49]$. Indeed, Gilman et. al. noted that the derivative thermogravimetric (DTG) curves obtained from the delaminated nylon-6/clay nanocomposites are almost identical to the values corresponding to pure nylon- 6 , whereas the peaks in the DTG of intercalated polystyrene/clay nanocomposites are shifted by $50{ }^{\circ} \mathrm{C}$ from the pure polymer [49]. The fact that the delaminated nylon-6/clay nanocomposites exhibit a reduction in flammability comparable to what is observed for the intercalated nanocomposites, even though the DTG results indicate that polymers are not stabilized when present in delaminated nanocomposites, suggests the possibility that the clay layers reorganize after some of the polymer is gasified. That is, the nanocomposite undergoes a phase change from a delaminated to an intercalated structure as a result of the change in composition brought 
about by the thermal degradation of the polymer. Once the intercalated nanocomposite is formed, it is stabilized by the mechanism discussed in the preceding paragraphs. This hypothesis is supported by transmission electron microscopy (TEM) of the char that remained after burning samples of the delaminated nylon-6/clay nanocomposites under a thermal flux of $35 \mathrm{~kW} / \mathrm{m}^{2}$ on the cone calorimeter. The TEM images clearly reveal an organized layered structure that was not present prior to combustion $[48,49]$.

\section{Time Averaged Rate of Mass Loss}

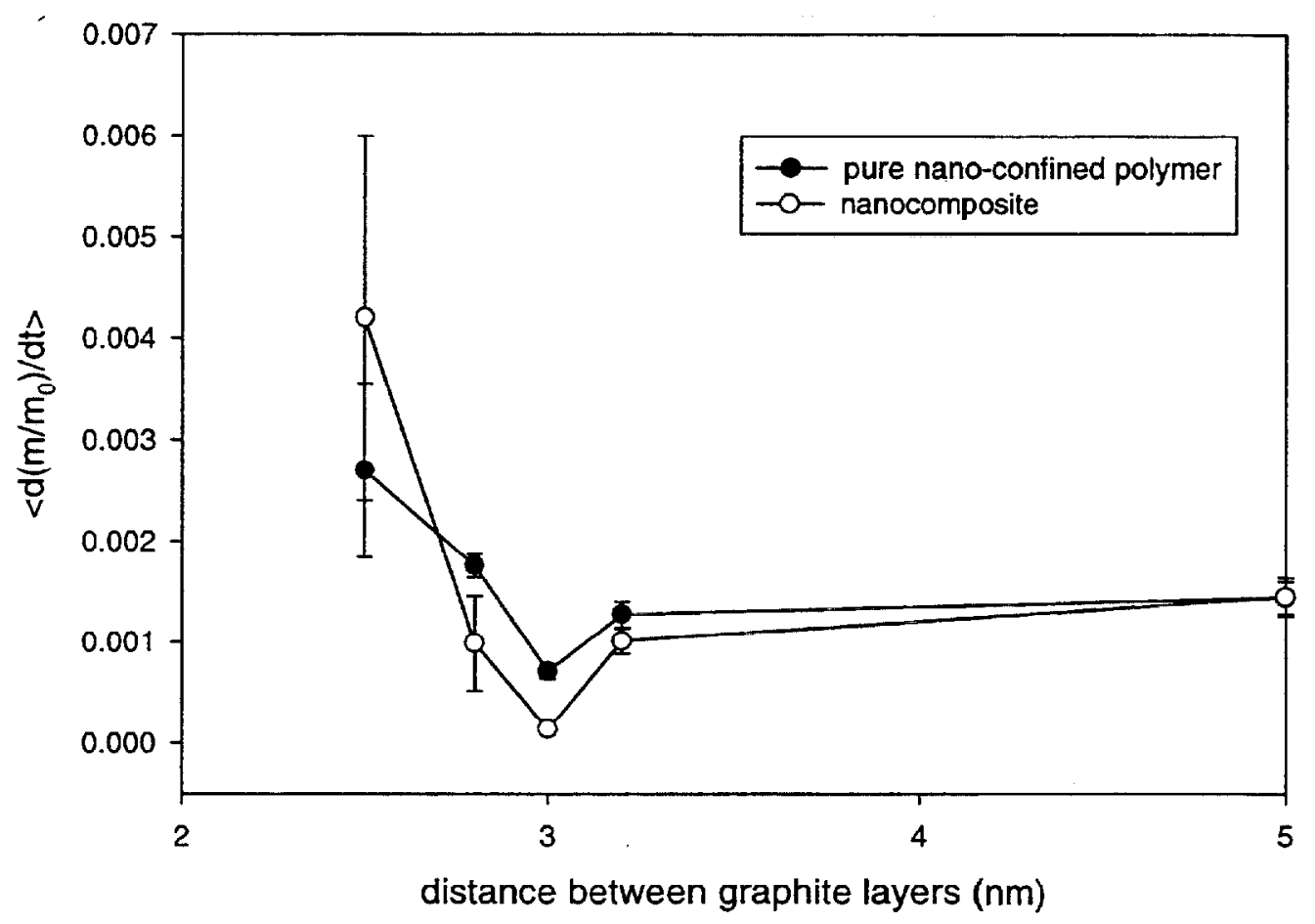

Figure 11. The average rates of mass loss from the nanocomposites (open circles) and non-interacting mixtures (closed circles) of the polymer and graphite as a function of the distance between the graphite sheets.

\section{2d Bromine-Containing Fire Retardant Additives}

A series of computer simulations were performed on polypropylene in the presence of some representative bromine-containing fire retardants. These included an aromatic compound, decabromobiphenyl, and two aliphatic hydrocarbons, tetrabromoethylene and a vinyl bromide oligomer. The purpose of this study was to make a qualitative comparison of the thermal degradation products and, thereby, to gain further insights into the nature of the mechanisms by which these additives affect a reduction in the flammability of the polymer. The procedures followed in this study were the same as described above in the 
case of the silica gel additives, except that all of the degrees of freedom, including the atoms that comprised the additives, were dynamic.

Bromine, in the form of $\mathrm{Br}, \mathrm{Br}_{2}$, and small gas phase hydrocarbons, was released early in the simulations. These products are apparent in Figure 12, which is a still frame from the simulated thermal degradation of a polypropylene blend containing both decabrombiphenyl and vinyl bromide oligomer. Additional simulations performed on the additives in the absence of the polymer indicated that $\mathrm{Br}_{2}$, as opposed to $\mathrm{HBr}$, is a major product of the thermal degradation of both decabromobiphenyl and tetrabromoethylene. This is in sharp contrast to what we observed in the simulated thermal degradation of the vinyl bromide oligomers. In this case, which should be representative of the general class of alkyl bromides, the major products were monomer and $\mathrm{HBr}$. Large amounts of atomic $\mathrm{Br}$ were also released, but the formation of molecular $\mathrm{Br}$ in the form of $\mathrm{Br}_{2}$ was not observed. The significance of this observation is that $\mathrm{Br}_{2}$ might be expected play a role in condensed phase suppression by abstracting hydrogens from polymer chains which then can cross-link to form a fire resistant char. Thus, based on standard tabulations of bond dissociation energies [39], the sequence of reactions consisting of the abstraction of 2 hydrogens by $\mathrm{Br}_{2}$ to form two molecules of $\mathrm{HBr}$ and a branched polymer is exothermic $(\Delta \mathrm{H} \sim-86 \mathrm{~kJ} / \mathrm{mol})$, whereas the analogous sequence involving the abstraction of hydrogen by $\mathrm{HBr}$ to form $\mathrm{H}_{2}+\mathrm{HBr}$ and a branched polymer is slightly endothermic $(\Delta \mathrm{H} \sim 10$ $\mathrm{kJ} / \mathrm{mol}$ ).

\section{Summary and Conclusions}

The various methods that comprise the field of molecular modeling were reviewed in the context of their potential for application to the molecular level design of new fire retardants and suppressants. The capabilities of these techniques were demonstrated by performing calculations on systems ranging in molecular complexity from small gas phase molecules to polymers.

An application of molecular quantum mechanics was considered first. These calculations were originally performed to assess the likely environmental impacts of a series of fluoro and chloro- methanes that were advanced as candidates to replace halon fire extinguishants. The computational strategy was based on a correlation between bond strengths and atmospheric lifetime. It was found that the $\mathrm{C}-\mathrm{H}$ bond dissociation energies for small gas phase molecules could be calculated to sufficient accuracy to warrant using this approach to screen replacement candidates. The further application of these methods in evaluating forcefield parameters for use in molecular mechanics, dynamics and Monte Carlo calculations on polymers was also discussed.

Next, the theory and implementation of a computer model of thermal degradation in polymers was presented. The predictive capability of the model was tested by 


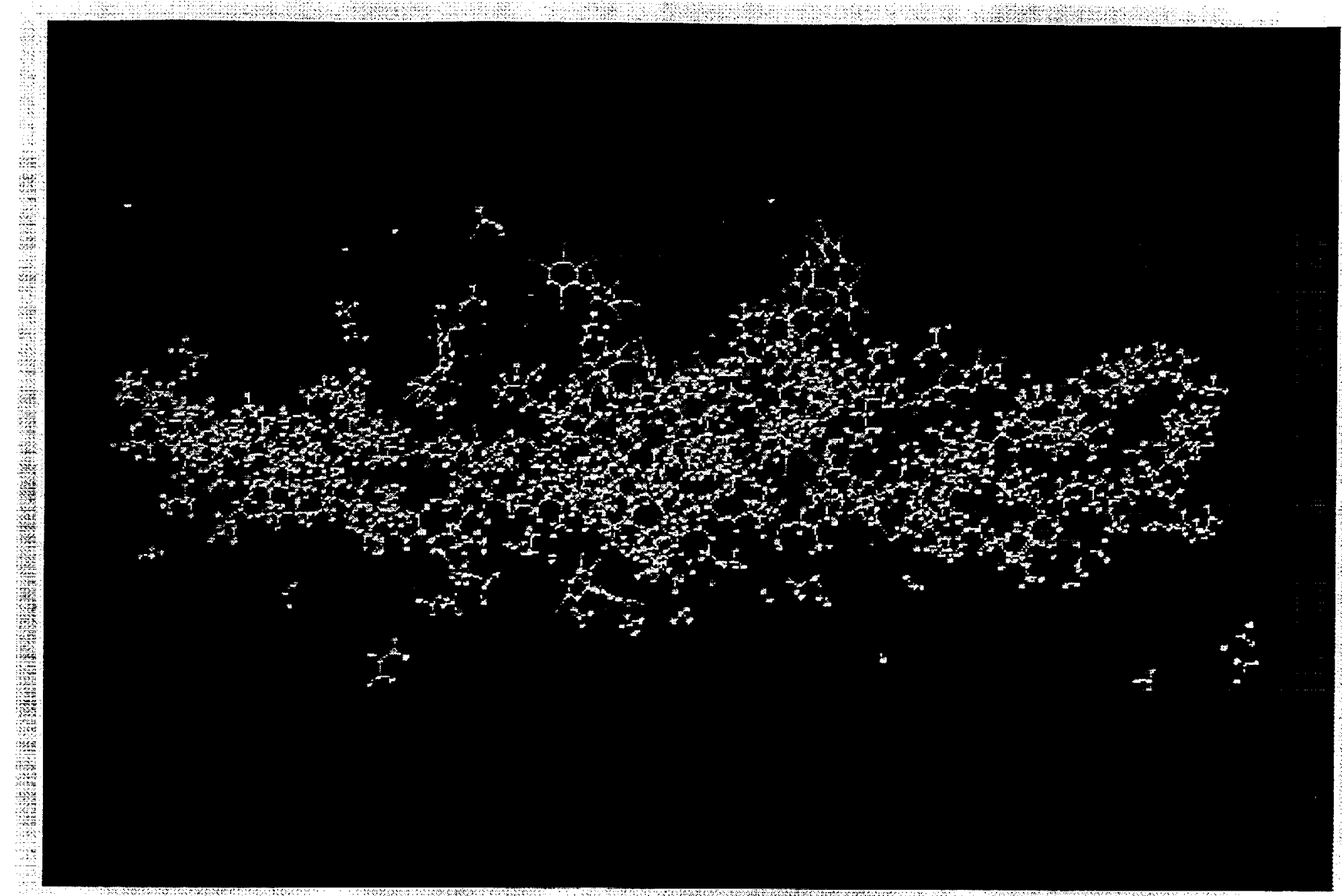

Figure 12. Thermal degradation of a blend of polypropylene with some brominated fire retardants.

comparing computed rate constants for beta-scission in a small gas phase molecule ( $\mathrm{n}$ pentane) to the experimental values for temperatures between $1000 \mathrm{~K}$ and $1600 \mathrm{~K}$. Although they are in reasonable agreement with experiment, the fact that the discrepancies exceed the uncertainty in the calculations at some temperatures suggests that there might be additional sources of error.

Finally, a series of calculations were performed to study the effects of fire retardant additives on the thermal degradation of polypropylene. Included in this survey were blends of polypropylene with silica gel, three representative bromine-containing additives, and a polypropylene/graphite layered nanocomposite. The results, although qualitative in nature, are consistent with experimental findings and, at the same time, provide new insights into the mechanisms by which these additives affect a reduction in the flammability of the polymer.

The applications presented in this chapter corroborate the premise that molecular modeling can be used to predict the properties of new materials in ways that macroscopic 
models cannot and, thereby, offers an unrivaled potential for computer aided molecular design. The major limitation of this approach is that the amount of computational effort required to obtain molecular level detail presently restricts the domain of these calculations to infinitesimal scales of time and distance, which limits their application to model systems. These boundaries, however, are receding as advances in computer technology enable calculations on more realistic materials. Based on the rapid pace of the progress attained so far, it seems likely that molecular modeling will soon become an integral part of industrial research and development programs in materials flammability.

\section{References}

1. G.L. Nelson. Recycling of Plastics - A New FR Challenge. The Future of Fire Retarded Materials: Applications \& Regulations. Lancaster, PA: FRCA, 1994, 135.

2. D. Lenoir and K. Kampke-Thiel. Formation of Polybrominated Dibenzodioxins and Dibenzofurans in Laboratory Combustion Processes of Brominated Fire Retardants. In: Fire and Polymers II. Materials and Tests for Hazard Prevention. G.L. Nelson, Ed. ACS Symposium Series 599, 1995, pp 377-392.

3. J.H. Krieger. Computational Chemistry. Chemical \& Eng. News 75:26-32, 1997.

4. E.K. Wilson. Computers Customize Combinatorial Libraries. Chemical \& Eng. News 76:31-37, 1998.

5. M.R. Nyden and D.W. Noid. Molecular Dynamics of Initial Events in the Thermal Degradation of Polymers. Phys. Chem. 95: 940-945, 1991.

6. M.R. Nyden, G.P. Forney and J.E. Brown. Molecular Modeling of Polymer Flammability: Application to the Design of Flame-Resistant Polyethylene. Macromolecules 25: 1658-1666, 1992.

7. M.R. Nyden, J.E. Brown and S.M. Lomakin. Molecular Dynamics Modeling of Polymer Flammability. Mat. Res. Soc. Symp. Proc. 278: 47-53, 1992.

8. M.R. Nyden, T.R. Coley and S. Mumby. Applications of Molecular Dynamics to the Study of Thermal Degradation in Aromatic Polymers; I. Polystyrene. Polym. Eng. Sci. 37, No. 9:1496-1500, 1997. 
9. M.R. Nyden and J.W. Gilman. Molecular Dynamics Simulations of the Thermal Degradation of Nano-Confined Polypropylene. Comp. and Theor. Polym. Sci., 7, 191-198 (1997).

10. E. Ranzi, M. Dente, T. Faravelli, G. Bozzano, S. Fabini, R. Nava, V. Cozzani, and L. Tognotti. Kinetic Modeling of Polyethylene and Polypropylene Thermal Degradation. J. Anal. Appl. Pyrolysis 40-41: 305,319, 1997.

11. K. M. Butler. Physical Modeling of Intumescent Fire Retardant Polymers. In: Polymeric Foams. K.C. Khemani, Ed. ACS Symposium Series 669, 1997, pp 214230.

12. M.R. Nyden, G.T. Linteris, D.R.F. Burgess Jr., P.R. Westmoreland, W. Tsang and M.R. Zachariah. Flame Inhibition Chemistry and the Search for Additional Fire Fighting Chemicals. In: Evaluation of Alternative In-Flight Fire Suppressants For Full-Scale Testing in Simulated Aircraft Engine Nacelles and Dry Bays. W.L. Grosshandler, R.G. Gann and W.M. Pitts, Ed. NIST SP 861, 1994, pp 467-641.

13. W.H. Press, B.P. Flannery, S. Teukolsky, and W.T. Vetterling. Numerical Recipes in C. The Art of Scientific Computing. Cambridge: Cambridge University Press, 1991, pp353-394.

14. A. Messiah. Quantum Mechanics. Vol. II. New York: John Wiley\& Sons, 1958, pp. $762-800$.

15. M. Born and J.R. Oppenheimer. Zur Quantentheorie der Molekeln. Ann. der Physik 389: 457-484, 1927.

16. K.K. Irikura and D.J. Frurip. Computational Thermochemistry. In: Computational Thermochemistry Prediction and Estimation of Molecular Thermodynamics. Washington DC: American Chemical Society, 1996, pp 2-18.

17. J.R. Maple, U. Dinur, and A.T. Hagler, Derivation of Force Fields for Molecular Mechanics and Dynamics from Ab Initio Energy Surfaces, Proc. Natl. Acad. Sci. USA 85: 5350-5354, 1988.

18. N.A. Metropolis, A.W. Rosenbluth, M.N. Rosenbluth, A.H. Teller, and E. Teller. Equation of State Calculations by Fast Computing Machines. J. Chem. Phys. 21: 1087-1092, 1953.

19. M. Guaita and O. Chiantore. Molecular Mass Changes the Thermal Degradation of Poly- $\alpha$-Methylstyrene. Poly. Deg. And Stability 11: 167-179, 1985. 
20. M. Guaita, O. Chiantore, and L. Costa. Changes in Degree of Polymerization in the Thermal Degradation of Polystyrene. Poly. Deg. And Stability 12: 315-332, 1985.

21. P. Dauber-Osguthorpe, V.A. Roberts, D.J. Osguthorpe, J. Wolff, M. Genest, A.T. Hagler. Structure and Energetics of Ligand Binding to Proteins: E. Coli Dihydrofolate Reductase-trimethoprim, a Drug-receptor System. Proteins: Structure, Function and Genetics 4:31-47, 1988.

22. J.L. Harrington. The Halon Phaseout Speeds Up. NFPA Journal March/April:3842, 1993.

23. J.T. Herron. Global Warming Potenial. In: Preliminary Screening Procedures and Criteria for Replacements for Halons 1211 and 1301. R.G. Gann, J.D. Barnes, S. Davis, J.S. Harris, R.H. Harris, Jr., J.T. Herron, B.C. Levin, F.I. Mopsik, K.A. Notarianni, M.R. Nyden, M. Paabo, and R.E. Ricker. NIST TN-1278, 1990, pp $121-172$

24. W.M. Pitts, M. Nyden, R.G. Gann, W.G. Mallard, and W. Tsang. Construction of an Exploratory List of Chemicals to Initiate the Search for Halon Alternatives. NIST TN-1279, 1990.

25. R.G. Gann, Ed. Fire Suppression System Performance of Alternative Agents in Aircraft Engine and Dry Bay Laboratory Simulations. NIST SP 890, 1995

26. R.E. Huie and V.L Orkin. Screening Methods for Agent Compatibility with the Global Environment. Screening Methods for Agent Compatibility with People, Materials and the Environment, Gaithersburg, MD, 1998.

27. Z. Zhang, R.D.Saini, M.J. Kurylo, and R.E. Huie. Rate Constants for the Reactions of the Hydroxyl Radical with Several Partially Fluorinated Ethers. J. Phys. Chem. 96: 9301-9304, 1992.

28. D.A. Fischer, C.H. Hales, D.L. Filkin, M.K.W. Ko, N.D. Sze, P.S. Connel, D.J. Wuebbles, I.S.A. Isaksen, and F.Stordal. Relative Effects on Stratospheric Ozone of Halogenated Methanes and Ethanes of Social and Industrial Interest. In: World Meteorological Organization, Global research and Monitoring Project - Report No. 20, Scientific Assessment of Stratospheric Ozone: 1989, 1989.

29. D.F. McMillan and D.M. Golden. Hydrocarbon Bond Dissociation Energies. Ann Rev. Phys. Chem. 33: 493-532, 1982.

30. DMOL User Guide. San Diego, CA: Biosym Technologies, Inc., 1992, pp. 8.18.32 . 
31. M.J. Frisch, G.W. Trucks, M. Head-Gordon, P.M.W. Gill, M.W. Wong, J.B. Foresman, B.G. Johnson, H.B. Schlegel, M.A. Robb, E.S. Repogle, R. Gomperts, J.L. Andres, K. Raghavachari, J.S. Binkley, C. Gonzalez, R.L. Martin, D.J. Fox, d.J. Defrees, J. Baker, J.J.P. Stewart, and J.A. Pople. Pittsburgh, PA: Gaussian Inc., 1992.

32. R.G. Parr and W. Yang. Density-Functional Theory of Atoms and Molecules. New York: Oxford University Press, 1989, pp 142-200.

33. R. Krishnan M.J. Frisch and J.A. Pople. Contribution of Triple Substitutions to the Electron Correlation Energy in Fourth-Order Perturbation Theory. J. Chem. Phys. 72: 4244-4245, 1980.

34. L.A. Curtiss, K. Raghavachari, G.W. Trucks, and J.A. Pople. Gaussian-2 Theory for Molecular Energies of First- and Second- Row Compounds. J. Chem. Phys. 94: $7221-7230,1991$.

35. R.J. Berry, D.R.F. Burgess, Jr., M.R. Nyden, M.R. Zachariah and M. Schwartz. Halon Thermochemistry: Ab Initio Calculations of the Enthalpies of Formation of Fluoromethanes. J. Phy. Chem. 99:17145-17150, 1995.

36. R.J. Berry, D.R.F. Burgess, Jr., M.R. Nyden, M.R. Zachariah and M. Schwartz. Halon Thermochemistry: Calculated Enthalpies of Formation of Chlorofluoromethanes. J. Phy. Chem. 100:7405-7410, 1996.

37. R.J. Berry, C.J. Ehlers, D.R.F. Burgess, Jr., M.R. Zachariah, M.R. Nyden and M. Schwartz. Halon Thermochemistry: Ab Initio Calculations of the Enthalpies of Formation of Fluoroethanes. J. Molec. Struct. (Theochem) 422:89-98, 1998.

38. K.K. Irikura, Systematic Errors in ab Initio Bond Dissociation Energies. J. Am. Chem. Soc. Submitted.

39. C.F. Cullis and M.M Hirschler. The Combustion of Organic Polymers. Oxford, England: Clarendon Press, 1981, pp. 93-228.

40. Biosym Solutions. 6, San Diego, CA: Biosym Technologies, Inc., 1995.

41. Discover User Guide, Part 3, San Diego, CA: Biosym/MSI, Inc., 1995. 
42. R.H. Boyd. The Relationship Between the Kinetics and Mechanism of Thermal Depolymerization. In: Thermal Stability of Polymers. New York: Marcel Dekker, 1970, pp. 47-89.

43. L.S. Kassel. The Kinetics of Homogeneous Gas Reactions. New York: The Chemical Catalog Company, 1932, pp. 93-113.

44. W. Tsang, J.A. Walker, and J.A. Manion. Single Pulse Shock Tube Study on the Decomposition of 1-Pentyl Radicals. $27^{\text {th }}$ Symposium on Combustion (Int'l). in press, 1998.

45. J.W. Gilman, T. Kashiwagi, S. Lomakin, J.D. Lichtenhan, A. Bolf and P. Jones. Char Enhancing Approaches to Flame Retarding Polymers. Additives and Modifiers for Polymers, in press, 1998.

46. Y. Kojima, A. Usuki, M. Kawasumi, A. Okada, Y. Fukushima, T. Kurauchi and O. Kamigaito. Mechanical Properties of Nylon 6-Clay Hybrid. J. Mater. Res. 8:1185$1189,1993$.

47. E. Giannelis. Polymer Layered Silicate Nanocomposites. Adv. Mater. 8: 29-35, 1996.

48. J.W. Gilman, T. Kashiwagi, S. Lomakin, J.D. Lichtenhan, P. Jones, E. Giannelis and E. Manias. Nanocomposites: Radiative Gasification and Vinyl Polymer Flammability. Proceedings of The 6th European Meeting on Fire Retardancy of Polymeric Materials, Lille, France, 1997.

49. J.W. Gilman, T. Kashiwagi and J.D. Lichtenhan. Nanocomposites: A Revolutionary New Flame Retardant Approach. SAMPE Journal 33:40-46, 1997. 
NIST-114 U.S. DEPARTMENT OF

(REV. 6-93) NATIONAL INSTITUTE OF STANDARDS AND TECHNOLOGY

ADMAN 4.09

MANUSCRIPT REVIEW AND APPROVAL

INSTRUCTIONS: ATTACH ORIGINAL OF THIS FORM TO ONE (1) COPY OF MANUSCRIPT

AND SEND TO: WERB SECRETARY, BUILDING B20, ROOM 125

TITLE AND SUBTITLE (CITE IN FULL)

The Molecular Level Design of Fire Retardants and Suppressants

CONTRACT OR GRANT NUMBER

AUTHOR(S) (LAST NAME, FIRST INITIAL, SECOND INITIAL

Nyden, Marc R..

LABORATORY AND DIVISION NAMES (FIRST NIST AUTHOR ONLY)

Building and Fire Research Laboratory, Fire Science Division

SPONSORING ORGANIZATION NAME AND COMPLETE ADDRESS (STREET, CTY, STATE, ZIP)

\begin{abstract}
PROPOSED FOR NIST PUBLICATION
JOURNAL OF RESEARCH (NIST JRES)

J. PHYS. \& CHEM. REF. DATA (JPCRD)

HANDBOOK (NIST HB)

SPECIAL PUBLICATION (NIST SP)

TECHNICAL NOTE (TN)
\end{abstract}

PROPOSED FOR NON-NIST PUBLICATION (CTTE FULLY):

Fire Retardancy of Polymeric Materials, Marcel Decker Publishing Company
(ERB USE ONLY)

\begin{tabular}{l|l|}
\hline ERB CONTROL NUMBER & DIVISION \\
G & \\
\hline PUBLICATIONS REPORT NULAER & CATEGOAY CODE \\
No. NISTIR 6275 & \\
\hline $\begin{array}{l}\text { PUBLICATIONDATE } \\
\text { JanUATY } 1999\end{array}$ & NO. PAINTED PAGES \\
\hline
\end{tabular}

\section{TYPE OF REPORT AND/OR PERIOD COVERED}

PERFORMING ORGANIZATION (CHECK $(X)$ ONE BOX)

X NIST/GATTHERSBURG

NIST/BOULDER

NISTIJILA

ABSTRACT (A 2000-CHARACTER OR LESS FACTUAL SUMMARY OF MOST SIGNIFICANT INFOAMATION. IF DOCUMENT INCLUDES A SIGNIFICANT BIBLIOGRAPHY OR LTTERATURE SURVEY, CITE IT HERE. SPELL OUT ACRONYMS ON FIRST REFERENCE.) (CONTINUE ON SEPARATE PAGE, IF NECESSARY.)

The present generation of fire retardant additives and fire resistant polymers has come under increasing public scrutiny because of the perception that they can contribute to environmental problems. This concern has spurred interest in the development of new and more "environmentally friendly" treatments to reduce polymer flammability. The emergence of this issue has occurred at a time when computer technology has opened new possibilities for the molecular level design of additives and materials. Indeed, recent work conducted in this laboratory has demonstrated that molecular modeling is a promising approach to the development of new fire retardant additives and fire resistant materials. In this chapter, the various methods that comprise the field of molecular modeling will be reviewed and the viability of using these techniques to identify factors which alter the thermal degradation chemistries of polymers in ways that effect a reduction in their flammability will be assessed. The purview of this exposition will be extended to include consideration of quantum mechanical calculations performed on a series of small gas phase molecules that were advanced as candidates for the replacement of halon fire extinguishants.

KEY WORDS (MAXIMUM OF 9; 28 CHARACTERS AND SPACES EACH; SEPARATE WTTH SEMICOLONS; ALPHABETIC ORDER; CAPTALIZE ONLY PROPER NAMES) computer models; dynamics; polypropylene, thermal degradation; unimolecular reactions

AVAILABILTT:

$\mathrm{X}$ UNLIMITED $\square$ FOR OFFICIAL DISTRIBUTION - DO NOT RELEASE TO NTIS

ORDER FROM SUPERINTENDENT OF DOCUMENTS, U.S. GPO, WASHINGTON, DC 20402

$X$ ORDER FROM NTIS, SPRINGFIELD, VA 22161
NOTE TO AUTHOR(S); iF YOU DO NOT WISH THIS MANUSCRIPT ANNOUNCED BEFORE PUBLICATION, PLEASE CHECK HERE. 